\title{
Criminologie
}

\section{Modèles de dangerosité : les contrevenants sexuels et la loi}

\section{Michael Petrunik}

Volume 27, numéro 2, 1994

Sexe et criminalité

URI : https://id.erudit.org/iderudit/017357ar

DOI : https://doi.org/10.7202/017357ar

Aller au sommaire du numéro

Éditeur(s)

Les Presses de l'Université de Montréal

ISSN

0316-0041 (imprimé)

1492-1367 (numérique)

Découvrir la revue

Citer cet article

Petrunik, M. (1994). Modèles de dangerosité : les contrevenants sexuels et la loi Criminologie, 27(2), 87-125. https://doi.org/10.7202/017357ar

\section{Résumé de l'article}

The history of dangerous offender legislation reveals a selective focus on few sexual and violent offenders many of whom are not demonstrably more dangerous than most of the offenders from the larger pool of sexual and violent offenders from which they are drawn. To the extent such legislation draws attention from routine frequently occuring forms of violence in favour of the violence of a few predatory offenders it is clearly problematic. Nonetheless, there are good reasons such legislation is likely to be retained and perhaps modified to place a greater emphasis on community protection. 


\section{MODĖLES DE DANGEROSITÉ : \\ LES CONTREVENANTS SEXUELS ET LA LOI ${ }^{1}$ \\ Michael Petrunik²}

The history of dangerous offender legislation reveals a selective focus on few sexual and violent offenders many of whom are not demonstrably more dangerous than most of the offenders from the larger pool of sexual and violent offenders from which they are drawn. To the extent such le gislation draws attention from routine frequently occuring forms of violence in favour of the violence of a few predatory offenders it is clearly problematic. Nonetheless. there are good reasons such legislation is likely to be retained and perhaps modified to place a greater emphasis on community protection.

\section{INTRODUCTION}

L'histoire du concept de dangerosité est longue et controversée. Malgré les critiques des défenseurs des libertés individuelles, la notion de dangerosité a toujours attiré les législateurs et les groupes de pression soucieux de protéger femmes et enfants des personnes déséquilibrées et des criminels dangereux (Petrunik, 1982). Depuis un certain temps, un nouveau modèle de dangerosité. le modèle de protection communautaire, retient l'attention de plusieurs juridictions. Dans les pages qui suivent, je ferai l'historique des approches les plus importantes du contrôle social : d'abord le modèle clinique. ensuite le modèle juridique et, troisièmement, le modèle de protection communautaire. Je décrirai ces modèles, en ferai une évaluation et donnerai des exemples de législations et pratiques en vigueur dans certaines juridictions d'Europe. d'Amérique du Nord et d'Australie. Finalement. j'examinerai la législation et la pratique actuelles, au Canada en matière de dangerosité, de même que les récentes réformes proposées, et $j$ 'en soulignerai les problèmes potentiels. Je conclurai avec une discussion des facteurs qui, aux yeux du public d'aujourd'hui. rendent si souhaitable ce modèle de protection communautaire de dangerosité.

1. Cette recherche est le résultat d'un contrat passé avec le Solliciteur général du Canada. Les opinions exprimés ici sont exclusivement les miennes. La traduction est de Traductions Brumar.

2. L'autcur est professeur au Département de criminologie de l'Université d'Ottawa, I Stewart Strect. Ottawa, Ontario, K1N 6N5 


\section{DÉFINITION DE LA DANGEROSITÉ}

La justice criminelle et les législations sur la santé mentale utilisent depuis longtemps le concept de dangerosité pour définir les personnes présentant un risque élevé de danger physique. psychologique ou moral pour eux-mêmes ou les autres. Ce concept offre plusieurs aspects intéressants. Premièrement, il renvoie, non pas aux actes ou omissions, mais à leurs auteurs. Deuxièmement, il vise les auteurs de certains crimes violents plutôt que de l'ensemble des actes pouvant être considérés comme violents. On considère généralement comme dangereuses les personnes ayant commis des crimes sexuels, particulièrement lorsque les victimes sont des enfants. On estime rarement dangereux les pyromanes ou les terroristes politiques. Troisièmement, le concept de dangerosité évoque un certain état qui prédispose l'individu à commettre certains actes violents. Les caractéristiques propres a l'individu, plus que les circonstances pouvant provoquer de tels actes, semblent constituer la source du danger. Quatrièmement, le concept de dangerosité regarde plutôt vers l'avenir. le passé d'un individu n'ayant d'intérêt que pour prévoir et contrôler sa conduite future.

\section{LE CONTEXTE SOCIAL DE LA LÉGISLATION SUR LES CONTREVENANTS DANGEREUX}

Toutes les sociétés ont des conceptions différentes du genre de personnes présentant un danger pour les autres. Par exemple, certaines catégories de personnes, comme les sorcières, les personnes possédées du démon, les malades mentaux, les agresseurs sexuels, les personnes souffrant de désordres de la personnalité (psychopathes et sociopathes) ont toujours représenté, dans l'histoire, une source exceptionnelle de danger. Ces personnes constituent un risque par rapport à certains types d'offenses (crimes violents et sexuels) et à certaines catégories de victimes (femmes et enfants). La législation criminelle touchait généralement quatre principales catégories de personnes à risque : agresseurs sexuels. individus violents, récidivistes et personnes considérées malades mentales ou présentant des troubles de personnalité. Ces catégories se recoupent plutôt que de s'exclure, avec le résultat que la législation sur la dangerosité a souvent visé d'abord les récidivistes violents réputés avoir une maladie mentale, un trouble de la personnalité ou une autre anomalie mentale.

L’attitude générale de la société face aux personnes considérées dangereuses est la peur, une peur incontrôlable devant une menace qui semble irrationnelle et imprévisible et devant une conduite physiquement et moralement repoussante ou anormale. La peur augmente lorsque la menace concerne de jeunes enfants, perçus comme les membres les plus innocents et les plus vulnérables de la société. Cette peur de la société s'exprime alors 
par des reportages détaillés et sensationnalistes renforçant les archétypes populaires (Best. 1990). En légiférant en matière de dangerosité, les sociétés ont toujours voulu d'abord se rassurer plutôt qu'aborder réellement le problème. Elles s'attaquent à des cas isoles, hautement publicisés, exceptionnels, atypiques, plutôt qu'à l'ensemble des cas. plus nombreux et moins spectaculaires. Malgré les recherches (Marshall et Vaillancourt. 1993: Statistique Canada, 1993; Abel et Rouleau, 1990) montrant que la violence ou l'agression sexuelle surviennent plus fréquemment dans un cadre familial ou parmi un groupe de personnes interreliées, les tois sur la dangerosité s'appliquent d'abord aux auteurs de violence et d'agression sexuelle étrangers. En certains cas, cette législation peut exclure les auteurs de violence domestique (État de Washington, 1989, 1991).

Les recherches sur la mise en application des lois sur la dangerosité (Scheingold, 1992; Petrunik, 1982; Sutherland, 1950a, 1950b; Swanson, 1960-1961: Tenney, 1962) montrent que l'adoption de telles lois répondait souvent à un incident sensationnel qui avait révolté la population et mené à la mobilisation de certains groupes exerçant des pressions sur les politiciens afin qu'ils adoptent des réformes. La législation sur la dangerosité apparaît đonc d'abord comme une tentative pour apaiser la colère et la crainte de la population et pour servir ensuite des intérêts personnels (par exemple des politiciens cherchant à se faire rélire; des professionnels de la santé mentale ou de la justice désireux d'obtenir des ressources supplémentaires). plutôt que comme un effort concerté visant à réduire le danger réel pour le public.

\section{MODÈLES DE DANGEROSITÉ}

La législation et la pratique sur la dangerosité visent plusieurs objectifs sociaux comme la protection de la société, la justice et le traitement ou la réhabilitation des personnes. Historiquement. on a connu trois modèles différents : le modèle clinique. le modèle juridique et le modèle de protection communautaire.

Le modèle clinique s'attache aux diagnostic, pronostic et traitement des problèmes mentaux et troubles de la personnalité, trois tâches exécutées par la psychiatrie, la psychologie clinique et le travail social clinique. Le modèle clinique considère que l'exécution de crimes sexuels et violents et la tendance à la récidive trouvent leur source dans une pathologie de l'individu qui le rend non responsable ou seulement partiellement responsable de ses actions. Dans cette perspective, les traitements cherchent à réduire les risques de récidive. Le modèle clinique s'oppose à toute forme de châtiment, mais, dans certains cas, afï de protéger à la fois le public et le criminel et faciliter le traitement, prévoit la réclusion pour une période 
indéterminée, selon la nature et la gravité du trouble et le risque présenté par l'agresseur.

Le modèle juridique essaie de tenir compte des revendications des défenseurs des libertés individuelles et réformateurs tout en imposant une punition juste aux individus coupables d'actes répréhensibles. On les considère comme responsables de leurs actions (c'est-à-dire juridiquement sains d'esprit) et ils méritent un niveau de punition basé sur la gravité de leur offense et leurs antécédents. avec parfois des circonstances atténuantes ou aggravantes. Entrent alors en jeu les principes de proportionnalité. de détermination. de parcimonie et d'égalité devant la loi. La sentence doit se baser sur ce que le contrevenant a fait, et non sur ce qu'il pourrait faire. Avec le modèle juridique, le concept de dangerosité s'applique donc davantage au criminel qu'au crime.

Le modèle de protection communautaire reflète les vues des mouvements pour la défense des droits des victimes, la prévention du crime et la protection des femmes et des enfants. D'après ces groupes, les législations et politiques issues des approches clinique et juridique n'ont pas réussi à protéger la société des dangers que présentent les contrevenants sexuels violents. Les porte-parole de la protection communautaire exigent des mesures de protection maximales pour le public, mesures qui peuvent, dans l'intérêt supérieur de la sécurité du public, limiter les droits des contrevenants. Pour les partisans de la protection communautaire, le traitement ou la réhabilitation des contrevenants ne doivent pas signifier une réduction de la supervision, réduction qui pourrait mettre en danger les femmes et les enfants.

Même si les trois modèles accordent des priorités différentes aux objectifs sociaux fondamentaux et que chacun a un objectif premier bien défini, en pratique ce n'est pas si simple. Le modèle clinique privilégie le traitement du contrevenant. mais il rencontre une forte opposition sociale désireuse de placer la protection de la société au-dessus du droit au traitement. Le modèle juridique accorde la primauté aux droits des contrevenants et malades mentaux, mais il doit faire face aux groupes qui accordent une importance égale ou supérieure aux droits des victimes et de leur famille et qui réclament aussi le droit à la sécurité pour la société. Le modèle de protection communautaire place au premier rang la sécurité des femmes et des enfants et une plus grande tranquillité pour le public. mais il ne fait pas non plus l'unanimité. On a contesté la constitutionnalité des lois récentes sur la protection de la société et plusieurs pensent qu'une incarcération illimitée sans réhabilitation efficace et sans mesures préventives pour contrer la violence ne règle pas le problème. 


\section{LE MODÈLE CLINIQUE : ORIGINES ET PRINCIPALES HYPOTHÈSES}

La notion de dangerosité, conçue comme une prédisposition au crime et aux activités antisociales, se retrouve déjà dans des écrits du XIX ${ }^{\mathrm{e}}$ siècle : certains textes de criminologie et de psychiatrie de l'époque parlent de "criminel psychopathe » ou de "criminel-né » et autres contrevenants présentant une forme $d$ ' «aliénation mentale» ou d' «imbécillité morale» (Rennie, 1978; Rieber et Vetter 1979; Werlinder 1978). On peut définir la psychopathie comme l'incapacité présumée d'un individu à manifester de l'empathie et du jugement moral; ce concept a contribué à développer la notion d'un certain type de contrevenant, réllement dangereux pour la société parce qu'il combine des tendances profondément enracinées, sinon innées, à poser des actes d'agression sexuelle, avec une absence d'empatbie et de sensibilitế morale.

Dans une publication de 1978, Michel Foucault traite de l'origine du modèle clinique, du diagnostic et du traitement du psychopathe criminel ou homme criminel et du danger qu'il présente pour la société. Foucault. citant le criminologue italien Garofalo, explique que la criminologie classique de DiBeccaria et Bentham identifie seulement deux éléments : le crime et le châtiment. L'école positiviste italienne d'anthropologie criminelle, contestant cette vision bidimensionnelle de la criminologie classique, a fait reconnaître l'existence de trois éléments : le crime, le châtiment et le criminel. Foucault note que le criminel de Garofalo n'est pas une personne qui a commis un crime, mais une "personne criminelle», une personne poussée par sa propre nature à commettre des crimes violents.

Une autre source du modèle clinique est le concept de «monomanie homicide», utilisé par les psychiatres du dix-neuvième siècle pour expliquer des crimes d'une grande brutalité ou de nature bizarre, sans motif apparent, comme le profit ou la passion, et sans relation avec les catégories psychiatriques connues à l'époque, telles l' «imbécillité », la « démence » ou la «fureur». L'insensibilité apparente de ces crimes semblait constituer une forme de folie non reconnue par la loi de l'époque qui innocentait et liberait les individus pour cause de folie.

D'après Foucault, les psychiatres pouvaient justifier leur droit d'intervention dans les cas de contrevenants «mentalement anormaux», mais juridiquement sains d'esprit, sur la base d'un modèle clinique privilégiant, non seulement le traitement individuel. mais aussi l'hygiène publique. Le psychiatre, se présentant comme diagnostiqueur et gardien de l'individu dangereux, jouait le rôle de protecteur public, exactement comme les médecins qui diagnostiquent et placent en quarantaine les personnes porteuses de maladies contagieuses (voir aussi Bellak. 1971). 
L'un des facteurs menant à l'acceptation juridique du modèle clinique de dangerosité a été le développement de la notion que les individus. peu importe leurs intentions premières, sont responsables de leurs actions du seul fait qu'ils ont créé un risque pour les autres. La législation et la pratique criminelles ont intégré cette notion par le biais de la notion de "personne criminelle» ou d' «état dangereux» (en français dans le texte) (Foucault. 1978, p. 17); d'après cette notion. on devrait baser le contrôle social, non pas sur la gravité du crime, mais bien sur la «capacité et la propension du contrevenant à commettre un crime» (Ancel, 1965. p. 15).

Dans le cas de contrevenants considérés comme dangereux et présentant des troubles mentaux ou de personnalité, le modèle clinique de dangerosité plaçait le devoir de l'État d'assurer la protection du public au-dessus du droit de l'individu à l'égalité devant la loi, à la liberté et à un procès juste: l'État, recourant à sa police et de ses pouvoirs parens patriæ, a le droit d'isoler et de traiter de tels contrevenants pendant des périodes de temps plus longues que pour certains autres crimes.

À la fin du XIXe siècle et au début du $\mathrm{XX}^{\mathrm{e}}$, avec la diffusion du modèle clinique de contrôle social chez les législateurs en justice criminelle et santé mentale, on a créé, à l'extérieur des structures régulières, des mesures spéciales pour les personnes dangereuses. En Europe, le modèle clinique de dangerosité se retrouve dans une variété de mesures visant les contrevenants sexuels et violents qui semblaient présenter un désordre mental ou un trouble de personnalité et les récidivistes dont la résistance aux punitions semble indiquer une forme analogue de pathologie. Ces mesures incluent les lois TBR des Pays-Bas passées en 1928, la loi de détention préventive du Danemark adoptée en 1925, et celle de la défense de la société de Belgique, adoptée en 1930 (Antilla, 1975; Evensen, n.d.: Mathiesen, 1965; Moyer, 1974; Sansone, 1976; Serrill, 1977b; Tulkens and Digneffe, 1979 ; Zagaris, 1977 ; The Netherlands Prison Service, n.d.).

En Amérique du Nord, les lois Sexual Psychopath. Sexually Dangerous Person et Defective Delinquency, adoptées entre 1930 et 1960 , définissent le modèle clinique de dangerosité. En 1938. l'Illinois a été le premier État américain à adopter certaines lois civiles spéciales d'internement pour psychopathes sexuels, lois qui venaient compléter la législation d'internement involontaire prévue au Code criminel et civil de l'État pour les personnes présentant des troubles mentaux. Vingt-cinq autres États et le District de Columbia ont suivi l'exemple de l'Illinois et adopté des législations analogues (Sleffel, 1977). Au Canada, le Code criminel compte, depuis 1948, une loi sur les crimes sexuels. Généralement, l'adoption de lois sur les psychopathes sexuels faisait suite à l'indignation du public au sujet de quelques incidents, et parfois d'un seul incident. comme le viol et le meurtre d'un enfant (Sutherland. 1950a. 1950b: Tenney, 1962). 
Les lois sur les psychopathes sexuels se ressemblent sur plusieurs points (Sutherland 1950a. 1950b; Tappan, 1950: Swanson, 1960-1961; Sleffel, 1977) :

1) Les contrevenants sexuels attaquent des étrangers plutôt que des membres de leur famille ou connaissances.

2) Les contrevenants sexuels commencent par des délits mineurs. comme l'«exhibitionnisme» ou le «voyeurisme», et passent graduellement a des actes plus graves, dont le viol et parfois le meurtre.

3) La tendance à commettre des délits d'ordre sexuel naît d'un trouble de la personnalité ou d'une anormalité mentale (psychopathie sexuelle) qui prédisposent l'individu atfecté à récidiver, malgré les sanctions pénales.

4) Les psychiatres et autres experts en santé mentale peuvent efficacement diagnostiquer les psychopathes sexuels et prévoir l'identité des récidivistes.

5) Le châtiment ne réussit pas à contrôler le psychopathe sexuel ; on doit plutôt avoir recours à l'internement prolongé et aux traitements ; $l$ 'indjvidu peut aussi éventuellement. par simple maturité naturelle, ne plus être considéré comme dangereux.

6) L'augmentation des délits d'ordre sexuel par des étrangers constitue une menace sérieuse à la société et ses représentants élus doivent réagir rapidement.

Sutherland (1950a et 1950b) soutient que les lois sur les psychopathes sexuels visaient, non pas à résoudre un problème social objectif, mais plutôt à servir les intérêts des experts en santé mentale en accentuant, sous prétexte de traitement, leur rôle d'agents de contrôle social. Sutherland (1950b, pp. 299,287) décrit la perspective clinique qui sous-tend de telles lois :

Les lois sur les psychopathes sexuels découlent [...] d'un mouvement social considérant les criminels comme des patients (p. 299).

Il y a eu tendance, non seulement vers l'individualisation du traitement des contrevenants, mais aussi vers des politiques psychiatriques. Le traitement se base sur la prémisse que le criminel est une personne socialement malade ; les causes du crime deviennent alors des déficiences que l'on considère permanentes et génériques (p. 287).

L'application du modèle clinique aux contrevenants sexuels a eu pour conséquence de représenter la dangerosité comme une pathologie individuelle. La dangerosité est alors devenue une figure rhétorique du diagnostic et du traitement de la maladie: les psychiatres et autres cliniciens devinrent 
les experts autorisés à se prononcer sur la dangerosité d'un individu et à recommander aux tribunaux, institutions pénales et autorités en santé mentale la détention ou la libération de ces individus.

\section{LE MODÈLE CLINIQUE DANS LA LÉGISLATION ET LA PRATIQUE ACTUELLES}

En Europe et Amérique du Nord, le modèle clinique a été très en vogue dans la législation criminelle et civile, du début du siècle jusqu'au début des années 1960. Depuis, on a aboli ou refondu en grande partie toutes ces législations. Certaines juridictions continuent à faire un grand usage de ce modèle clinique néanmoins, dont les Pays-Bas. l'Angleterre et le Pays de Galles, et l'Illinois.

Les Pays-Bas utilisent une clause du code criminel appelée TBS (une modification des lois TBR de 1928) dans le cas de contrevenants irresponsables en tout ou en partie de leur crime. à cause d'un désordre mental ou d'un trouble de la personnalité, et que l'on considère dangereux. La TBS s'applique au moment de la sentence de deux façons possibles : soit comme une alternative à la prison. soit comme une mesure qui s'applique après la fin d'une sentence déterminée. La TBS reste en vigueur pendant deux ans, mais est renouvelable indéfiniment pour les contrevenants violents considérés comme dangereux (Derks, et al. . 1993; Van Emmerik, 1993; Gouvernement des Pays-Bas, 1992 : Tigges, 1990 ; Koenraadt. 1990).

L'Angleterre et le Pays de Galles, avec leur Loi sur la santé mentale. utilisent un ordre de l'hôpital pour détenir indéfiniment des contrevenants atteints de maladie mentale et considérés comme dangereux. Cet ordre peut entrer en vigueur après un verdict d'insanité criminelle; la Cour peut l'appliquer à des contrevenants sains d'esprit présentant des désordres mentaux ou à un prisonnier purgeant une peine et chez qui on découvre un trouble mental (England et Wales, 1987 ; Baker, 1993)

Comme aux Pays-Bas, l'Angleterre et le Pays de Galles considèrent un trouble psychotique comme un trouble mental. L'ordre de l'hôpital ne peut mener à la détention que des contrevenants violents et sexuels souffrant d'un trouble psychotique ou d'une autre forme de désordre mental. Cet ordre est donc une mesure qui vise les contrevenants dangereux souffrant d'un désordre mental, et non tous les contrevenants dangereux.

La décision de libérer des personnes détenues par un ordre de l'hôpital relève du ministre de l'Intérieur ou du Tribunal de révision de santé mentale. Le patient ne doit pas souffrir d'un désordre mental nécessitant une hospitalisation, et l'absence de traitement en milieu clos ne doit pas menacer la sécurité du public ni la sienne propre. 
On a exprimé des réserves quant à l'utilisation des hôpitaux en ce qui a trait aux contrevenants psychopathes. Certains affirment que les contrevenants psychopathes, étant plus mauvais que fous, pourraient recevoir un traitement plus adéquat en prison qu'en milieu hospitalier. On croit toutefois que les psychopathes présentent un risque accru de récidive par rapport aux autres catégories de déficients mentaux. Les hospitalisations prolongées semblent donc offrir une plus grande protection pour le public qu'une sentence déterminée. premièrement à cause des traitements (même si le taux de réussite est très faible) et. deuxièmement, parce qu'on peut prolonger l'internement de façon indéfinie (Peay, 1988).

Le Illinois Sexually Dangerous Persons Statute (SDPS) prévoit l'internement indefini des contrevenants sexuels souffrant de troubles mentaux pendant une période d'au moins un an. L'État doit ou condamner et punir un individu accusé $d^{\prime}$ un crime sexuel, ou l'interner et le traiter: il ne peut faire les deux. La période statutaire d'internement est indéfinie. La Cour libérera, en tout temps, toute personne qui, d'après elle, ne remplit plus les critères du SDPS. On a critiqué le SDPS parce qu'il permet d'interner. selon des standards civils de preuve, des individus ne satisfaisant pas au critère «au-delà de tout doute raisonnable» du code criminel (Fujimoto, 1992 ; Grabowski, 1988; Temborius, 1977; Nangle, 1976; Burick, 1968).

\section{CRITIQUES DU MODÈLE CLINIQUE DANS LA LÉGISLATION ET LA PRATIQUE ENTOURANT LA DANGEROSITÉ}

Les critiques les plus fréquentes du modèle clinique ont été historiquement (1) la circularité de certains de ses concepts clés; (2) la faible fiabilité des diagnostics de désordre de personnalité : (3) l'imprécision des prédictions de comportements violents: (4) le faible taux de succès des programmes de traitement.

1) On a souvent critiqué la nature circulaire et vague de termes comme "désordre anti-social de la personnalité » et "psychopathe». On a appliqué ces catégories à des délinquances sexuelles et d'autres comportements antisociaux et on les a traitées comme la cause ou la source de ces comportements (Hakeem. 1958; Cirali, 1978)

2) Les diagnostics de désordres de la personnalité ne sont pas vraiment fiables. Dans des essais du DSM-III visant à obtenir un diagnostic de personnalités antisociales, des professionnels se sont trouvés en désaccord plus de la moitié du temps. Et ce pourcentage augmentait encore pour les autres catégories de désordres de la personnalitê (Wettstein, 1992, p. 604).

3) Il existe une documentation abondante sur les difficultés de prédire avec succès quels individus sous examen auront par la suite un comportement violent (Monahan, 1981, 1984b. 1988; Webster, 1990a et 1990b). Des 
recherches faites en grande partie auprès de déficients mentaux, et à leur insu, indiquent deux fois plus de prédictions de violence de la part de faux positifs que de prédictions de violence de vrais positifs. Les médias, exploitant l'indignation du public. n'hésitent pas à faire du sensationnalisme avec le petit nombre de faux négatifs. La réaction du public aux faux négatifs, même si leur occurrence est très faible, est à ce point forte que les cliniciens font preuve de beaucoup de réserve dans leur diagnostic (Steadman. 1972; Wettstein, 1992).

Les principaux problèmes associés aux prédictions de violence sont les suivants : les critères vagues des catégories de personnes dont on essaie de prédire le comportement; le manque à prendre en considération de la fréquence d'un comportement particulier à la fois dans une population donnée (par exemple les contrevenants sexuels) et dans certains sous-groupes de cette population (par exemple les pédophiles et violeurs); l'omission d'inclure des informations sur le contexte entourant ces comportements violents; la plus grande imprécision des prédictions à long terme par rapport aux prédictions à court terme : le type de comportement prédit (par exemple violent, comme le viol, ou non violent, comme l'exhibitionnisme) (Wettstein, 1992, pp. 606-608).

4) L'ensemble des critiques du modèle clinique de dangerosité portent sur la question du traitement. Le traitement semble avoir des effets perceptibles sur certaines catégories de psychoses. Toutefois, la probabilité de succès avec les contrevenants présentant des troubles de personnalité apparaît faible (Wettstein, 1992, p. 609 ; Harris et Rice, 1991).

Les programmes de traitement des contrevenants sexuels semblent aussi jusqu'à maintenant peu efficaces (Wormick et Borzecki, 1985; Furby et al., 1989). Une étude du programme de traitement des contrevenants sexuels de l'État de Washington, en 1985, a montré que le taux de récidive des contrevenants ayant suivi le programme équivalait à celui des contrevenants incarcérés sans traitement. Si l'on inclut les personnes qui ont abandonné le programme, le taux de succès du groupe admis au traitement est plus bas que celui du groupe sans traitement (Wettstein 1992, p. 608). Récemment, une étude de la Californie. en cours jusqu'à aujourd'hui (Sex Offender Treatment and Evaluation Project), a comparé des contrevenants traités et non traités; après cinq ans de suivi. l'étude montre. pour un programme très coûteux pour les contribuables (Scheingold, 1992, p. 815), des taux de récidive sensiblement identiques.

\section{L'AVENIR DU MODÈLE CLINIQUE}

L'efficacité du modèle clinique quant au diagnostic, à la prédiction et à la réhabilitation semble donc plutôt faible. 
Certains aspects nouveaux, toutefois, permettent un peu d'optimisme.

Les récents travaux théoriques et empiriques sur la psychopathie (Hart, Kropp et Hare 1988: Hare, Mcpherson et Forth 1988; Harris. Rice et Cormier 1991) semblent avoir résolu le problème de circularité des premières définitions de la psychopathie. En particulier, l'échelle de psychopathie de Hare (PCL et PCL-R) s'avère utile dans l'évaluation des contrevenants susceptibles de réhabilitation et des risques de récidive qu'ils présentent.

Dans l'évaluation de la dangerosité, on peut obtenir une certaine amélioration en distinguant des sous-groupes sur la base du diagnostic clinique et des antécédents, en combinant des techniques cliniques et actuarielles et en utilisant les informations contextuelles. et non plus seulement les caractéristiques du contrevenant. Même s'il est peu probable que de telles améliorations puissent résoudre le problème de la haute fréquence de faux positifs et même s'il y aura toujours de faux négatifs (Monahan, 1981, 1984, 1988: Wettstein, 1992), plusieurs cliniciens pensent qu'on peut espérer un certain succès, si mince soit-il.

Dans le domaine du traitement des contrevenants sexuels, on a récemment mis au point des approches intégrant une importante composante cognitive-comportementale. Ces approches visent, non pas à guérir le contrevenant, mais à prévenir la rechute (Laws, 1989 ; Pithers, 1990 ; Marshall et Barrett, 1990). Les premiers résultats du programme semblent assez prometteurs pour qu'on finance son développement et son évaluation.

\section{LE MODÈLE JURIDIQUE : LA DÉMYSTIFICATION DE LA DANGEROSITÉ}

Dans les années 1960 et 1970 , la philosophie du contrôle social a délaissé l'approche axée sur la sécurité du public pour favoriser une approche mettant l'accent sur la primauté des droits individuels. Des mouvements sociaux dénonçant l'inefficacité et les abus de la psychiatrie institutionnelle (Szasz, 1965: Stone, 1975), les inégalités créés par le système judiciaire et son incapacité à réduire la criminalité et à réhabiliter les contrevenants ont réclamé de plus en plus le respect des droits des prisonniers et malades mentaux. L'influence du modèle clinique diminuant, on constata l'émergence. à la fois en santé mentale et dans le système judiciaire, du modèle juridique qui mettait l'accent sur les droits fondamentaux. l'égalité devant la loi et l'utilisation réduite de contrôles restrictifs.

Le modèle juridique de sentence et sanctions (Fogel, 1975) repose sui la prémisse que tous les contrevenants sains d'esprit, incluant les contrevenants sexuels et violents, sont responsables de leurs actes et méritent d'être châtiés pour leurs crimes. Le modèle juridique s'intéresse d'abord au crime 
plutôt qu'au contrevenant. La sentence doit être proportionnelle à la gravité du crime commis et aux antécédents du contrevenant. Contrairement au modèle clinique qui réclame une sentence indéterminée ou une périnde de traitement en se basant sur la pathologie du contrevenant et les dangers qu'il présente pour l'avenir, le modèle juridique prévoit des sentences à durées fixes. Dans le modèle juridique, les droits individuels. l'égalité devant la loi et l'option la moins restrictive passent avant la protection de la société et la réhabilitation du contrevenant.

À mesure que le modèle juridique s'est imposé, on a essayé de démystifier le modèle clinique de dangerosité et la compétence des experts chargés de définir la dangerosité. Les individus considérés comme dangereux sont apparus comme les victimes des tendances conservatrices des cliniciens qui exagéraient les risques de violence présentés par les déficients mentaux et contrevenants sexuels (Steadman, 1972). Les défenseurs des droits individuels, soucieux de protéger les droits constitutionnels des contrevenants et déficients mentaux (Harvard Law Review, 1975; Chandler et Rose. 1973), contestèrent la notion de dangerosité comme base pour restreindre la liberté.

La remise en question des concepts cliniques a été un autre thème important de la démystification du modèle clinique de dangerosité. On a d'abord dit que le concept de psychopathie n'était que l'expression médicale d'un jugement moral sur la méchanceté humaine (Hakeem, 1958; Bleechmore, 1975; Cirali, 1978). Cette critique de la notion de psychopathie a amené plusieurs États à changer le nom des lois; la loi Sexual Psychopathy est ainsi devenue la loi Sexually Dangerous Persons. Et comme pour s'éloigner davantage de la rhétorique clinique, on s'est mis à utiliser le terme contrevenant dangereux. comme dans les lois canadiennes Dangerous Sexual Offender (1960) et Dangerous Offender (1977). Cet usage moins fréquent de l'expression "psychopathe sexuel » reflétait l'opinion que les contrevenants sexuels étaient responsables de leurs actes et méritaient châtiment. Dans ce contexte, le traitement passe après la condamnation (Weisberg, 1984).

Le second thème majeur de la démystification du modèle clinique de dangerosité a été l'habileté accrue des cliniciens à prédire la violence des individus considérés comme dangereux. Dans une étude sur les prédictions de violence par des experts en santé mentale. Monahan (1981) a montré que les prédictions de la violence se réalisaient seulement dans un cas sur trois. Monahan mentionne le suivi de personnes libérées sur ordre de la Cour après violation de leurs droits constitutionnels. Dans les études Baxstrom de l'État de New York (Cocozza et Steadman, 1976 et 1978), au moins deux individus sur trois, libérés sur ordre de la Cour contre l'avis des médecins, n'ont pas récidivé pendant une période de suivi de quatre ans. On 
a noté les mêmes phénomènes en Pennsylvanie et au Massachussetts (Thornberry et Jacoby. 1977: MacGarry et Parker, 1974). La fréquence élevée des «faux positifs» est devenue, particulièrement pour les défenseurs des droits individuels, la preuve que la plupart des contrevenants présumément dangereux ou déficients mentaux n'étaient pas vraiment dangereux et que les présumés experts en santé mentale n'étaient pas les experts que l'on croyait.

Un troisième thème important de la démystification du modèle clinique de la dangerosité a été l'émergence d'un mouvement pour la défense des libertés individuelles et droits constitutionnels. En citant des recherches montrant la grande imprécision des évaluations de dangerosité et la faible efficacité des traitements pour les personnes incarcérées en vertu des lois sur la psychopathie sexuelle, les défenseurs des libertés individuelles ont réussi à faire abolir ces derniêres lois et à mettre fin à l'internement indéterminé et au traitement involontaire. À travers les États-Unis, on s'éloigna des lois sur la psychopathie sexuelle et, plus généralement, des sentences indéterminées (Serril, 1977c; McGee, 1978). En 1977, le Maryland a aboli sa loi Defective Delinquency (Contract Research Corporation, 1977) et, en 1982. la Californie a aboli sa loi Mentally Disordered Sexual Offender (Oliver, 1982). En 1981, l'État de Washington a adopté sa loi Sentencing Reform Act, qui, en se basant sur une combinaison des condamnations courantes et passées, établissait un éventail standard de sentences pour tous les crimes. La Cour ne pouvait alors s'écarter des sentences standards que si elle découvrait des circonstances aggravantes ou atténuantes. Le Sentencing Reform Act est entré en vigueur pour tous les crimes après le $1^{\text {er }}$ juillet 1984. De plus, en 1984, l'État de Washington, modifiant sa philosophie de sentence, a aussi aboli sa loi sur la psychopathie sexuelle. et appliqué. pour tous les crimes, des sentences déterminées plutôt qu'indéterminées. Les contrevenants sexuels faisaient maintenant face à un éventail standard de sentences et la participation à un programme de traitement devenait volontaire (État de Washington, 1989).

En passant du modèle clinique au modèle juridique, on a mis en sourdine le traitement du criminel. les droits des victimes et la sécurité du public au profit des principes fondamentaux de justice et des droits individuels des contrevenants et déficients mentaux. Par conséquent. on a contesté les lois sur la psychopathie sexuelle et les personnes sexuellement dangereuses sur la base que ces lois violaient les principes fondamentaux de la justice, incluant l'égalité devant la loi, le droit d'accepter ou de refuser le traitement, le principe de proportionnalité et celui de l'option la moins restrictive (Wald et Friedman, 1978). À preuve, on cita de nombreux cas d'abus des droits individuels, par exemple des personnes non dangereuses détenues beaucoup plus longtemps que prévu par la loi criminelle (Szasz, 1977). 
La remise en question du modèle clinique de dangerosité a provoqué, non seulement un mouvement vers des sentences fixes dans le système judiciaire, mais aussi l'introduction d'une plus grande détermination dans l'internement des déficients mentaux (Stone, 1975). À moins d'avoir été trouvés non coupables pour raison d'insanité ou d'incapacité à subir un procès, tous les contrevenants, sexuels et violents. étaient considérés suffisamment raisonnables et responsables de leurs actions pour recevoir une punition. Tous ces contrevenants méritaient aussi la pleine protection de la loi. Le modèle juridique rejetait surtout les lois spéciales permettant l'internement indéfini des contrevenants sexuels dont on ne pouvait prouver la déficience mentale. Pour les défenseurs du modèle juridique, réclamer une plus grande protection de la société par des moyens cliniquement justifiables était non seulement philosophiquement intenable. mais constituait aussi une violation de droits fondamentaux, sinon sacrés.

\section{CRITIQUES DU MODÈLE JURIDIQUE}

Si le modèle juridique reprochait au modèle clinique de ne pas protéger les droits des contrevenants, on a reproché au modèle juridique de ne pas sauvegarder la sécurité du public. De plus, les critiques du modèle juridique ont contesté la façon dont ses défenseurs citent des recherches critiquant le modèle clinique et soutenant leur propre approche. On en a conclu que les modèles clinique et juridique comportaient des défauts sérieux parce qu’ils oubliaient de sauvegarder la sécurité du public.

1. Les recherches démontrant le peu de fiabilité des évaluations de dangerosité (particulièrement la haute fréquence des faux positifs) ont été faites avec des populations de malades mentaux trouvés non coupables pour aliénation mentale ou incapacité à subir leurs procès. On ne peut projeter les résultats obtenus avec ce groupe sur des groupes de contrevenants sexuels et violents qui n'ont jamais rencontré les critères les déclarant officiellement malades mentaux (Brooks, 1992, pp. 747-748).

2. Même si les prédictions cìniques surestiment le nombre de faux positifs. ce nombre n'est peut-être pas aussi élevé que les défenseurs du modèle juridique le prétendent. L'utilisation des statistiques de condamnations des contrevenants sexuels, par exemple, peut diminuer la dangerosité (Groth et Longo. 1982; Brooks, 1992, pp. 744-746) pour plusieurs raisons :

(i) Le taux de dénonciation des agressions sexuelles ou tentatives d'agressions sexuelles est très faible (Brickman et Brière, 1989; Lizotte, 1985: Abel et al., 1987: Wright, 1984; Polk, 1985). Une enquête canadienne sur les victimes en milieu urbain a découvert que $62 \%$ des temmes victimes d'agressions sexuelles ne rapportaient pas cette agression à la police (Solliciteur général du Canada, 1985). Le comité sur Les crimes 
contre les enfants et les jeunes (1984) indique pour sa part que les trois quarts des répondants féminins et les neuf dixièmes des répondants masculins ne rapportaient pas l'agression dont ils avaient été victimes à une personne en autorité. Plus récemment, un sondage téléphonique de Statistique Canada (1993) auprès de 2300 femmes de 18 ans et plus a montré qu'on rapportait à la police seulement $14 \%$ des incidents violents et seulement $9 \%$ à des services sociaux.

(ii) La dénonciation des crimes sexuels ne mène pas souvent, à cause de difficultés techniques, à des accusations ni à des poursuites. Par exemple, la Cour refuse souvent de tenir un procès faute de preuves, ou parce que la victime refuse de témoigner ou qu'on ne peut assurer la sécurité des témoins (Marshall et Barbaree, 1990, p. 378).

3. Les sentences dans le cas des contrevenants sexuels sont souvent non proportionnelles aux torts causés. On sous-estime souvent le tort causé par les contrevenants sexuels aux femmes et aux enfants, même s'il n'y a pas eu de sévices corporels. Les recherches montrent qu'il peut y avoir un tort psychologique se manifestant de nombreuses annees après le crime. Par ailleurs. l'utilisation de telles preuves présente un problème, car une partie, sinon une grande partie, du tort causé à la victime relève autant du traumatisme de l'incident que de la façon dont les membres de la famille, la police, les tribunaux et services sociaux traitent cet incident (Finkelhor, 1986; Hanson, 1990; Kendall-Tackett et al., 1993).

4. L'approche sentencielle du modèle juridique prêterait le flanc aux critiques. particulièrement dans le cas des contrevenants sexuels récidivistes. Une étude canadienne récente (Direction de la recherche et des statistiques. Services correctionnel du Canada. 1991, p. 5) sur l'ensemble des contrevenants dans les prisons fédérales montre que :

Comparés à tous les contrevenants sexuels. les contrevenants sexuels récidivistes (qui ont déjà purgé une peine de prison pour un crime sexuel) sont deux fois plus susceptibles de commettre un autre crime sexuel (et) beaucoup plus susceptibles de violer leurs conditions de liberation conditionnelle.

De plus, des recherches indiquent que certaines catégories de contrevenants sexuels, particulièrement les agresseurs d'enfants, présentent un très haut risque de récidive. Une étude de Hanson portant sur 197 agresseurs d'enfants, condamnés entre 1965 et 1973 . a découvert que $42 \%$ d'entre eux subissaient une autre condamnation pour un crime violent ou sexuel au cours de périodes de suivi allant de 19 à 28 ans. Même si les cinq à dix années qui suivent la libération semblent la période la plus risquée presque le quart des récidivistes ont été condamnés pour un nouveau crime sexuel. 
plus de dix ans après leur libération (Direction de la recherche et des statistiques. Service correctionnel du Canada. 1993, pp. 7-10).

Robinson (1989. p. 13) a assuré un suivi de l'étude de Barbaree et Marshall portant sur 170 hommes traités pour pédophilie et supervisés pendant une période de quatre ans. Utilisant les dossiers de la Cour, de la police et de Children's Aid, les chercheurs ont découvert un taux de récidive de $20.7 \%$. Lorsqu'on ajoute les sources non officielles de la police et de Children's Aid, ce chiffre grimpe à presque $60 \%$ de récidive. Un nombre important de ces crimes ont été commis par un conjoint, un parent. un autre membre de la maisonnée ou un ami de la famille, par opposition à un pur étranger.

\section{LE MODÈLE JURIDIQUE ET LE MODÈLE CLINIQUE EN PERSPECTIVE}

Au cours des années 1960 et 1970 , le modèle juridique de contrôle social a exercé une grande influence sur la législation en matière de dangerosité, de sorte que plusieurs juridictions des États-Unis ont aboli ou révisé de façon draconienne les lois basées sur le modèle clinique (lois sur la psychopathie sexuelle). On a noté une tendance analogue dans les pays nordiques (Svendsen, 1977: Petrunik, 1982). Dans sa version la plus stricte, le modèle juridique prétend que tous les contrevenants, sauf les aliénés, doivent se soumettre aux mêmes critères de sentence. Cela a mené à l'abolition de la sentence indéterminée et à l'introduction d'une variété de sentences déterminées (Serrill, 1977c). Le modèle juridique a aussi influencé les juridictions qui n'avaient pas aboli la sentence indéterminée : on a changé la terminologie (passant de «psychopathe sexuel» à «personne dangereuse » ou «contrevenant dangereux») ou certaines décisions de la Cour ont exigé une protection égale pour les personnes intimées en vertu des lois de dangerosité civile ou criminelle.

Même si le modèle juridique protège les droits des contrevenants et des malades mentaux et encourage le respect des droits fondamentaux. il n'a rien fait pour calmer la peur du public au sujet des contrevenants sexuels. En fait, le pendule est passé du pôle de la protection des droits individuels à celui de la protection du public. On a donc exigé un nouveau modèle de contrôle social s'attaquant sérieusement au problème du risque que présentent les contrevenants violents.

\section{LE GLISSEMENT DU MODÈLE JURIDIQUE VERS LE MODÈLE DE LA PROTECTION COMMUNAUTAIRE}

Le modèle juridique désirait sauvegarder les droits civils des contrevenants et malades mentaux, droits souvent bafoués dans le modèle clinique. Ce faisant, le modèle juridique aurait tendance à négliger la sêcurité du 
public et les droits des victimes réelles et futures. Comme le dit Monahan (1984, p. 12) :

La justice, dans le sens le plus large du terme, doit considérer, non seulement les effets de la sentence sur les contrevenants pour le crime commis, mais aussi sur les personnes innocentes qui seront les prochaines victimes des récidivistes.

À la fin des années 1980, en Amérique du Nord et en Australie, un modèle de protection communautaire a fait son apparition afin de répondre à ces attentes. Le souci majeur de ce modèle est la menace que les agresseurs sexuels laissent planer sur les membres vulnérables de la société. particulièrement les femmes et les enfants. Plusieurs groupes sociaux ont participé à l'élaboration du modèle de protection communautaire. Au niveau de la population, on a assisté à la naissance de mouvements pour la défense des victimes et pour la prévention du crime. et le public en général a exigé un plus grand respect de la loi et de l'ordre. Ces mouvements vont de pair avec les groupes pour la défense des femmes et des enfants qui s'inquiètent des dangers et menaces auxquels doivent faire face ces femmes et enfants, particulièrement les agressions sexuelles. Au niveau politique, le gouvernement, en réponse à ces revendications de la population, a mis l'accent sur la loi et l'ordre plus que sur des politiques orientées vers la réhabilitation et la sécurité publique (Scheingold, 1984, 1993).

Le modèle de protection communautaire s'articule autour des points suivants :

(1) Les contrevenants sexuels et violents représentent un danger réel et sérieux pour les femmes et les enfants. Même si le nombre de ces contrevenants est relativement petit. ils sont la cause de sévices physiques et psychologiques importants.

(2) Les politiciens et bureaucrates ont accordé trop d'attention aux droits des contrevenants et pas assez aux victimes des crimes violents et sexuels et à leur tamille. On a négligé les questions de sécurité du public en matière de crimes violents.

(3) Le faible taux de succès des programmes de réhabilitation et de traitement des contrevenants sexuels et violents signifie qu'à leur libération, ils constituent encore un grand danger pour le public. Les contrevenants sexuels et violents devraient rester enfermés jusqu'à ce qu'ils ne représentent plus de danger pour le public.

(4) Les instances juridiques et médicales n'ont pas réussi à surveiller adéquatement les individus dangereux libérés. De plus, ces deux systèmes fournissent une information insuffisante sur ces individus, de sorte que le public n'adopte pas ou n'est pas en mesure d'adopter des mesures de protection. 
Au Canada, aux États-Unis, en Australie et en Europe. la couverture médiatique intensive de crimes violents et sexuels horrifiants a nourri la peur du public. Ces actes ont été commis par des hommes possédant une longue histoire criminelle, juridique et médicale. hommes qu'on avait libérés même si, aux yeux de certaines autorités juridiques et médicales. ils constituaient une menace sérieuse. Dans certains cas. la loi forçait ces libérations parce que ces personnes avaient purgé leur sentence et ne remplissaient pas les critères d'internement involontaire de la loi sur la santé mentale.

Certains exemples sensationnels, comme le viol et la mutilation sexuelle d'un garçonnet de sept ans par Earl Shriner dans l'État de Washington (Boerner, 1992), les menaces et tentatives de meurtre par Gary David a Victoria, Australie (Fairall, 1993), et l'enlevement, le viol et le meurtre de Christopher Stephenson, un garçonnet de onze ans, par Joseph Fredericks en Ontario, Canada (Ontario, Ministère du Solliciteur général, bureau du chef-coroner, 1993), ont provoqué une levée de boucliers en faveur de l'adoption de lois pour la protection du public.

\section{LA LOI DE PROTECTION COMMUNAUTAIRE DE L'ÉTAT DE WASHINGTON}

Le Community Protection Act de l'État de Washington, qui inclut les clauses controversées Sexually Violent Predators. constitue un exemple d'approche de protection communautaire de la dangerosité.

L'indignation populaire devant plusieurs incidents hautement publicisés a été la principale cause de l'adoption de cette loi. L'indignation du public a été à son comble après le viol et la mutilation, en 1989, d'un garçonnet de sept ans, par Earl Shriner, un homme légèrement retardé. d'apparence physique bizarre, possédant un dossier d'agressions sexuelles sadiques mais une seule condamnation. Au moment du crime, Shriner circulait sans supervision parce que sa sentence était terminée et qu'un juge avait statué que. en vertu des lois sur la santé mentale de l'État, il ne remplissait pas les critères de «danger imminent» nécessaires à l'internement. L'incident Shriner a amené la création d'un groupe de défense des victimes appelé Brigade du soulier de tennis, nom suggéré par le soulier de tennis pour enfant qui a mené la police à la découverte du garçonnet violé et mutilé. Le soulier de tennis est devenu le symbole de tous les enfants à la merci des contrevenants sexuels. Un deuxième groupe. Les Amis de Diane. a été fondé par la mère de la jeune fille violée et assassinée par Gene Kane. un contrevenant sexuel libéré dans le cadre d'un programme de travail (Boerner, 1992, pp. 534-538). 
Le lobbying des groupes de défense de ces victimes a eu pour conséquence la création d'un Task Force on Community Protection (Gouvernement de l'État de Washington. 1989), qui comptait dans ses rangs les mères des deux victimes. En 1990. l'État de Washington, suivant les recommandations du groupe spécial (Task Force), a adopté une loi-cadre exhaustive. The Community Protection Act. conçu pour calmer l'inquiétude grandissante provoquée par les contrevenants sexuels. Parmi ces mesures, on trouve (Latond, 1992, p. 655):

(1) l'expansion de la liste des crimes sexuels afin d'inclure l'effraction de domicile et la pyromanie avec motif sexuel:

(2) une plus grande sévérité des sanctions pour la plupart des crimes sexuels :

(3) le traitement obligatoire des contrevenants sexuels mineurs:

(4) la prolongation de la période de supervision d'après libération dans le cas de certains criminels ;

(5) une obligation pour les criminels conđamnés et libérés de se rapporter à la police:

(6) la divulgation d'informations sur les allees et venues des criminels :

(7) un avis au procureur de la Couronne au moins 90 jours avant la libération ;

(8) finalement. la mesure la plus controversée : la clause d'internement civil indéterminé, après la fin de la sentence, pour les contrevenants sexuels considérés comme des prédateurs sexuels violents.

La loi Sexually Violent Predators visait à combler les lacunes issues des réformes du début des années 1980, qui avaient aboli les sentences criminelles indéfinies, le traitement involontaire pour les contrevenants sexuels et les mesures d'internement civil pour les psychopathes sexuels. pour les remplacer par des sentences d'emprisonnement à durée déterminée et le traitement volontaire.

Ces réformes n’avaient pas prévu qu’après avoir purgé leur sentence. les contrevenants sexuels se retrouvaient libres, sans supervision, alors que. dans plusieurs cas, ils avaient participé sans succès à un programme de traitement ou avaient tout simplement refusé toute forme de traitement. Les clauses de psychopathie sexuelle ne permettaient plus l'internement involontaire. Le système civil de santé mentale ne prenait soin que des cas extrêmes de maladies mentales. et non des désordres de la personnalité comme la psychopathie. L'internement involontaire n'était possible que dans les cas où le critère de «danger imminent» entrait en jeu. Il s’ensuivit alors une situation où des personnes considérées comme très dangereuses ont recouvré la liberté (Boerner, 1992. pp. 542-544). 
Selon David Boerner, membre du groupe spécial du gouverneur de l’État de Washington et principal rédacteur de la loi Sexually Violent Predator, cette loi ne se voulatit pas un modèle pour les autres juridictions (Boerner, 1993). Elle voulait simplement prévenir un problème dans l'État même de Washington, c'est-à-dire calmer l'indignation et la peur du public devant l'inefficacité apparente du système judiciaire. Boerner a soutenu que le principe moteur de cette loi aurait protégé le public d'Earl Shriner. l'homme qui a violé et mutilé un garçonnet de sept ans et. de ce fait, provoqué la colère générale et mené a la création du groupe spécial du gouverneur (Boerner, 1993).

L'attaque brutale de Shriner a eu lieu en mai 1989. Même s'il avait une longue histoire de violence grave. Shriner n'avait été condamné qu'une fois. À l'âge de seize ans, après avoir tué une adolescente de quinze ans, Shriner a été interné à titre de «contrevenant déficient», mais sans être trouvé coupable d'un crime. Entre 1977 et 1987, il a purgé une sentence de dix ans de prison sans libération conditionnelle pour avoir enlevé et violé deux adolescentes de seize ans. À l'expiration de sa sentence, on a tenté sans succès de l'interner civilement, malgré la découverte de plans détaillant la manière dont il enlèverait, séquestrerait et torturerait ses victimes. Entre 1987 et son arrestation en 1989. Shriner a purgé deux sentences de comté, une pour agression et l'autre pour séquestration illégale. Au moment de son arrestation pour enlèvement et agression sexuelle, Shriner attendait son procès pour une autre accusation (Boerner, 1992, pp. 526-529, 544$545)$.

Le groupe spécial du gouverneur a conclu que Shriner s'était glissé dans les interstices des systèmes judiciaire et médical et que des gens comme lui présentaient un tel danger qu'on devait sceller ces interstices. Plutôt que d'avoir recours à une législation large. comme le Sexual Psychopath Act, qui pourrait, en principe, s'appliquer à la plupart des agresseurs sexuels, le groupe spécial a opté pour un point de vue plus restrictif. se concentrant sur un petit nombre d'agresseurs sexuels considérés comme très dangereux sur la base de leur Jossiers judiciaires el médicaux. Bien qu'on prétendait alors identifier moins de faux positifs et améliorer, par rapport au Sexual Psychopath Act. l'équilibre entre les libertés individuelles et la protection du public, cette loi visait d'abord à calmer l'indignation populaire et à rassurer les gens (Boerner, 1993).

Avec les clauses Sexually Violent Predator du Community Protection Act entrées en vigueur le $1^{\text {er }}$ juillet 1990 , l'État de Washington a établi une procédure prévoyant qu'un individu ayant purgé une sentence pour au moins un crime sexuel violent pouvait devoir se soumettre à une évaluation déterminant s'il était prédateur sexuel violent. Les SVP définissent toute personne condamnée précédemment ou actuellement accusée d'un ou 
plusieurs crimes de violence sexuelle, considerée comme mentalement anormale ou déséquilibrée, ce qui la rend susceptible de récidiver. Les SVP $n$ 'incluent pas les agresseurs membres de la famille ni les connaissances de la victime. à moins de pouvoir prouver que l'agresseur a entretenu une relation avec la victime afin de l'agresser. Une personne trouvée coupable d'être un prédateur sexuel violent au cours d'un procès devant juge ou jury est internée indéfiniment au centre d'internement spécial de l'État. sans clause de libétation conditionnelle (Quinsey, 1992). La libération ne peut survenir qu'avec une décision de la Cour, après procès devant juge ou jury, statuant que l'agresseur ne remplit plus les critères pertinents.

La loi sur les prédateurs sexuels est en vigueur depuis quatre ans. Jusqu'en février 1993. 20 personnes avaient rempli les critères et la Cour en avait fait interner 10 (Weston, 1993). On a contesté la constitutionnalité de la loi en arguant que cette loi violait les droits fondamentaux des personnes internées. On a invoqué le droit à être libéré d'une punition cruelle et exagérée et le droit à l'égalité devant la loi (Bodine, 1990; Gleb, 1991; Greenlees, 1991 ; Lafond et Kagan, 1992; Lafond, 1992, Summers, 1991). Récemment, la Cour suprême de l'État de Washington a toutefois confirmé la constitutionnalité de cette loi par un vote de 6 à 3 .

À Victoria, en Australie, on a proposé un autre exemple de protection communautaire, le Community Protection (Violent Offenders) Bill, visant à remplacer la loi de détention préventive (la «loi Gary David») qui voulait protéger le public contre un individu dangereux en particulier. La nouvelle loi s'applique aux agresseurs violents qui ont purgé une peine privative et que l'on considère encore comme dangereux. Cette loi vise toute personne dont le dossier révèle au moins une hospitalisation psychiatrique involontaire et qui, d'après deux psychiatres, présente un sérieux desordre de la personnalité le rendant hautement susceptible de commettre un autre crime contre la personne (Victoria, Australie, 1990, 1991, 1992).

Comme la loi de l'État de Washington, la loi de Victoria a essuyé des critiques, du point de vue du modèle juridique, parce qu'elle prône une détention préventive qui viole plusieurs droits fondamentaux (Fairall. 1993).

\section{LÉGISLATION ET PRATIQUE SUR LA DANGEROSITÉ AU CANADA}

L'histoire de la législation et de la pratique sur la dangerosité au Canada commence par l'émergence de lois basées sur le modèle clinique, continue par une critique de ces lois du point de vue du modèle juridique avec seulement quelques modifications et se poursuit au cours des dernières années par une évolution vers un modèle de protection communautaire. 
En 1947 et 1948, on a intégré les mesures sur le Contrevenant d'habitude (Habitual Offender) et sur le Contrevenant psychopathe sexuel (Criminal Sexual Psychopath) au Code criminel. En 1960, on a amendé la mesure sur le Contrevenant psychopathe sexuel pour la remplacer par celle du Contrevenant sexuel dangereux (Dangerous Sexual Offenders). En 1977. la loi sur le Contrevenant dangereux a abrogé les lois sur le Contrevenant d'habitude et le Contrevenant psychopathe sexuel. La nouvelle loi s'appliquait encore aux contrevenants sexuels dangereux, mais permettait aussi d'inclure les agresseurs dangereux coupables de crimes violents. mais non sexuels.

En décembre 1992, en vertu des dispositions sur les Contrevenants dangereux, 121 personnes purgeaient des peines de prison de durée indéterminée. De plus, on a déclaré plusieurs individus contrevenants dangereux, tout en les condamnant à des peines de durée déterminée (Shore, 1984, pp. $419-420$ ). On trouve presque la moitie ( 59 ou $48.8 \%$ ) des contrevenants dangereux condamnés en Ontario, un peu plus du quart (32 ou $26,4 \%$ ) en Colombie-Britannique, et le quart restant dans les provinces de l'Atlantique et les Prairies. Le Québec ne compte pas d'individus déclarés contrevenants dangereux (Ministère de la Justice, 1993, pp. 21, 24).

Une proportion importante $(24 \%)$ des contrevenants considérés dangereux ne sont pas de race blanche, ce qui dénote peut-être la présence d'un préjugé contre les contrevenants issus de minorités. Certains observateurs (Berzins, 1983, p. 3; Webster et Dickens. 1983, p. 109; Esses et Webster, 1988 ) croient que l'apparence bizarre ou inusitée de certains contrevenants considérés comme dangereux peut avoir influencé le jugement.

Très peu de recherches portent sur l'application de la loi et le processus du jugement (Webster et Dickens, 1984; et al., Webster, 1987). Il semble toutefois que la Couronne utiliserait la loi sur les contrevenants dangereux comme argument lors de négociations visant une réduction de peine (Shore, $1988: 418$; Webster et Dickens, 1984, p. 109).

Plus de la moitié des contrevenants dangereux ont été reconnus coupables d'un délit sexuel et $90 \%$ d'entre eux comptent un délit sexuel ou plus à leur dossier. (Ministère de la Justice. 1993. pp. 24-25). Les individus déclarés contrevenants dangereux constituent seulement une petite proportion des contrevenants violents et contrevenants sexuels tels que définis par les dispositions de la loi. Prenons l'exemple des contrevenants sexuels. Au 31 octobre 1992, on comptait 1814 contrevenants (14.5\% de la population des établissements de détention fédéraux) coupables d'un délit sexuel (Ministère de la Justice, 1993, p. 20). Le recensement national des contrevenants sexuels indique qu'une majorité de ces contrevenants choisissent leurs victimes parmi les enfants ou les adolescents : en effet. $43,6 \%$ des cas 
concernent des victimes «adultes seulement», les enfants ou adolescents étant victimes dans plus de la moitié des cas.

Considérant le petit nombre de personnes déclarées formellement dangereuses (un peu plus de 120 sur une période de 15 ans), la réticence de certaines provinces (notamment le Québec) à se prévaloir du classement des contrevenants dangereux et le grand nombre d'individus présumés remplir les critères de la loi. la loi ne contribue pas vraiment à protéger le public. De plus, la loi sur les contrevenants dangereux n'a pas mené au développement de formes efficaces d'intervention clinique, que ce soit au point de vue du diagnostic. de la prédiction ou du traitement.

\section{LÉGISLATION ET PRATIQUE SUR LA DANGEROSITÉ APRÈS 1977 : LA NAISSANCE DU MODĖLE DE PROTECTION COMMUNAUTAIRE}

Avec une moyenne de seulement 8 nouveaux cas par année, la loi canadienne sur les contrevenants dangereux offre peu de réconfort au public effrayé par le risque élevé d'agression sexuelle et violente. Depuis le début des années 1980, en réponse à la demande du public et de certains groupes de pression, et en réaction à la couverture médiatique sensationnaliste de certains incidents de violence sexuelle, le gouvernement a adopté toute une série de lois et pratiques de dangerosité.

Le premier développement d'après 1977 en législation et pratique correctionnelle touchant la dangerosité est survenu après deux incidents isolés d'agression sexuelle et meurtre, commis en 1981, par Paul Kocurek et Duane Taylor, deux agresseurs sexuels libérés sous supervision obligatoire. Le fait que les deux victimes étaient des enfants a particulièrement excité la fureur du public (Marshall et Barrett, 1990, pp. 24-25).

Comme les clauses de rémission de la Loi sur les libérations conditionnelles prévoyaient la libération presque automatique des prisonniers ayant purgé les deux tiers de leur sentence, le Conseil national des libérations conditionnelles a instauré la pratique du «bouchon» (gating). On libérait donc le prisonnier avec supervision obligatoire et on émettait immédiatement un mandat d'arrestation suspendant la supervision obligatoire en prétendant que le prisonnier présentait un tel risque de récidive qu'on devait le ramener en prison. Après 11 cas de «bouchon», la Cour suprême s'est prononcée contre cette pratique en 1983 en arguant que, sans lois précises autorisant la suspension de la supervision obligatoire avant la liberation. une telle suspension devait se baser sur la conduite après la libération (Gouvernement du Canada, 1993, p. 3).

En 1984, le viol et meurtre de Cecelia Ruygrok, une jeune employée d'une maison de réinsertion sociale, par Allan Sweeny, criminel en libération conditionnelle, a amené la présentation de la loi C-67, en 1985. 
L'adoption de cette loi, en 1986, a permis au Conseil national des libérations conditionnelles de retenir prisonnier, au-delà de la date de sa libération obligatoire, un individu condamné pour un crime violent s'il y avait une présomption raisonnable que cet individu pouvait, avant l'expiration du mandat, commettre un crime causant un tort sérieux ou la mort (Gouvernement du Canada, 1993, p. 14 ; Marshall et Barrett, 1990, pp. 32-34).

En 1987, un an après l'adoption de la loi C-67, le viol et meurtre de Tema Conter par Melvin Stanton, agresseur sexuel en congé temporaire (Marshall et Barrett, 1993, p. 32) a provoqué une nouvelle flambée d'indignation populaire. Après des débats intenses et du lobbying, on a adopté, en 1992. la loi C-36 régissant le système correctionnel et la mise en liberté sous conditions. Cette loi privait du droit à la libération sur parole accélérée et au programme de congé temporaire non escorté tous les agresseurs violents et sexuels à haut risque, reportait l'admissibilité à la libération sur parole d'un sixième de la sentence à 6 mois avant l'admissibilité à la libération sur parole entière, et repoussait la détermination à l'admissibilité individuelle à la libération conditionnelle du tiers de la sentence à la moitié (Loi C-36, 1992; Gouvernement du Canada, 1993).

Tout au long de 1992, les médias ont continué à monter en épingle les cas impliquant des attaques contre les femmes et les enfants et à souligner le besoin de découvrir de nouvelles façons de protéger les membres vulnérables de la société. Le Conseil des libérations conditionnelles a ainsi relâché Wray Budreo, pédophile chronique comptant 23 accusations d'agressions sexuelles depuis 1963 et purgeant à ce moment-là une peine de six ans de prison pour trois crimes incluant le soudoiement de jeunes pour les inciter à des attouchements sexuels sur lui-même. En vertu de la Loi C-36, Budreo avait droit à la liberté après les deux tiers de la sentence, à moins qu'il ne soit susceptible de commettre un tort grave. Toutefois, l'opposition du public fut tellement forte que le Conseil, basant sa décision sur de nouvelles preuves de la dangerosité de Budreo, a renversé sa décision et exigé de Budreo qu'il purge sa sentence complète (Appleby, 1992; Viennau, 1993).

La plus forte réaction du public, toutefois, est venue en réponse à l'enquête du coroner de l'Ontario sur la mort, en 1988. de Christopher Stephenson, un garçonnet de 11 ans enlevé, violé et assassiné par Joseph Fredericks, un agresseur sexuel considéré comme un pédophile sadique et psychopathe. Au moment du meurtre, Fredericks était sous supervision obligatoire après avoir purgé les deux tiers d'une peine de cinq ans pour avoir violé un autre garçon de 11 ans. Fredericks aurait sans doute été classé contrevenant dangereux, mais la famille de la victime a voulu s'éviter l'épreuve de témoigner, ce qui amené à une peine réduite de cinq ans et à l'abandon des procédures de classement. 
L'enquête Stephenson, de l'automne 1992 jusqu'à l'hiver 1993, a reçu la couverture nationale des médias (Hudson, 1993; Sarick, 1993). En tout, l'enquête a présenté 71 recommandations dont. par exemple, une plus grande sévérité de la justice et des institutions de santé mentale.

La première recommandation était la «création d'une législation qui protégerait le public en permettant la détention des prédateurs sexuels violents au-delà de la fin de leur sentence ou d'autres conditions de détention telles qu'autorisées par le Code criminel du Canada, de même la participation à un programme de traitement pendant la détention ». On suggérait le Community Protection Act (Ontario, Ministry of the Solicitor General, 1993, p. 8) de l'État de Washington comme modèle de législation.

Avant même les recommandations de l'enquête Stephenson, le ministère fédéral du Solliciteur général faisait des études internes et revoyait ses programmes et politiques afin de trouver des réponses à ce qui était devenu une question politique et publique très controversée.

En mai 1993, alors que le parti progressiste-conservateur était au pouvoir, le Solliciteur général du Canada, Doug Lewis, a proposé une série de mesures touchant les contrevenants violents et sexuels récidivistes à haut risque et visant à répondre aux recommandations de l'enquête Stephenson. Dans le communiqué de presse commentant les mesures (Solliciteur général du Canada, 25 mai 1993), M. Lewis soulignait le besoin de ces mesures, dans le cadre d'un modèle de protection communautaire :

Les cas de violence des récentes années indiquent un besoin de nouveaux moyens pour traiter avec le groupe de contrevenants qui. même peu nombreux, n'en sont pas moins très dangereux et que nous ne pouvons pas réhabiliter ni détenir au-delà de leur sentence.

Le public en a assez d'un système qui libère des contrevenants violents présentant un haut risque de récidive. Le public demande avec raison où sont ses droits, où sont les droits des victimes, particulièrement les enfants, à ne pas se faire violer ou tuer par ces contrevenants.

[...] Le consensus est clair parmi les Canadiens : le gouvernement doit pouvoir détenir ces contrevenants violents tant et aussi longtemps qu'ils constituent une menace pour la société.

Les propositions du gouvernement conservateur (Ministère du Solliciteur général, 1993) contenaient les mesures suivantes :

1. une révision du Code criminel et de la Loi C-36 afïn de permettre le classement de contrevenant dangereux dans le cas de contrevenants purgeant déjà une sentence définie pour une agression grave contre la personne. Sur avis du Service correctionnel du Canada, le Conseil national des libérations conditionnelles aurait le pouvoir de proposer un classement 
possible de contrevenant dangereux pour un prisonnier. On ferait une telle recommandation au procureur général de la province où le contrevenant a reçu sa dernière sentence pour agression grave contre la personne. La recommandation devrait satisfaire quatre critères :

(a) une sentence courante pour une agression grave contre la personne: (b) un ordre de détention jusqu'à la fin de la sentence basé sur la probabilité d'une agression pouvant causer la mort ou des blessures graves: (c) une décision du Conseil national des libérations conditionnelles sur la probabilité, après la fïn de la sentence, d'une agression pouvant causer la mort ou des blessures graves; (d) des preuves de dangerosité non présentées devant la Cour qui a condamné le contrevenant pour une ou plusieurs agressions graves contre la personne.

Si les quatre critères sont satisfaits aux yeux du procureur général de la province, le classement de contrevenant dangereux pourrait entrer en vigueur au cours de la dernière année de la sentence. Dans le cas où la fïn de la sentence surviendrait avant une décision sur le classement de contrevenant dangereux, le procureur général pourrait s'adresser à la Cour afin de maintenir le contrevenant sous les verrous jusqu'à ce que la décision soit prise.

Dans le cas de classement officiel comme contrevenant dangereux, il y a quatre possibilités : (a) la détention interminée; (b) une période déterminée de détention: (c) la liberté surveillée pendant une période de dix ans; (d) la détention et la liberté surveillée. Le Service correctionnel du Canada administrerait les ordres de détention et la liberté surveillée. L'admissibilité à la libération sur parole arriverait un an après la date de l'ordre, avec révision de la demande chaque année subséquente ;

2. l'élimination du critère "dommages graves» pour une «agression sexuelle sur mineur» afin de pouvoir envoyer les agresseurs sexuels au Conseil national des libérations conditionnelles qui décidera s'ils doivent purger leur peine complète. Les critères de révision seraient une sentence courante de deux ans ou plus pour une agression sexuelle contre un enfant et la probabilité raisonnable d'une autre agression sexuelle contre un enfant avant la fin de la sentence.

Même si, par des changements dans la pratique sentencielle et correctionnelle, la loi vise à protéger le public contre les contrevenants violents et agresseurs sexuels, la mise en pratique de ces politiques a suscité d'autres problèmes. Un débat s'est engagé sur l'opportunité pour la police d'informer le public des allées et venues des agresseurs sexuels (Rogers, 1993a). Un sondage téléphonique Angus Reid-Southam News auprès de 1501 adultes canadiens, publié le 27 février 1993, indique que $69 \%$ des répondants souhaitaient la divulgation par les médias des noms des agresseurs à haut risque libérés de prison (Bindman, 1993, p. A3). Devant le vide 
juridique ou l'absence de politique formelle. plusieurs services de police de l'Ontario ont pris l'initiative de communiquer aux médias les noms des agresseurs sexuels (Rogers, 1993b; Richardson. 1993a).

\section{IMPLICATIONS DES LOIS ET DE LA PRATIQUE DANS D'AUTRES JURIDICTIONS AU CANADA}

L'examen historique de la législation et de la pratique dans plusieurs juridictions met en relief la variété des options que les sociétés ont à leur disposition pour aborder le problème des agresseurs sexuels et violents, que ce soit par l'entremise du système judiciaire et des institutions de santé mentale ou de quelque système hybride conçu pour prendre soin de ceux qui se glissent entre les deux premiers. Cet examen révèle aussi que la législation et la pratique sur la dangerosité au Canada ne peuvent se modeler sur celles des autres juridictions. parce que. par exemple. une telle législation se base souvent sur une interprétation différente de la responsabilité criminelle et des liens entre dangerosité et troubles de la personnalité. En Angleterre, au Pays de Galles et aux Pays-Bas, les clauses sur le contrevenant dangereux sont plus intimement liees au diagnostic de trouble mental et à la détermination de la responsabilité criminelle qu'au Canada. Au contraire, la jurisprudence canadienne et la pratique en médecine légale ne lient pas explicitement la dangerosité à une responsabilité diminuée causée par une psychopathie ou d'autres formes de troubles de la personnalité. L'approche canadienne de la dangerosité épouse davantage les soucis de sécurité publique de l'État de Washington. Toutefois, l'utilisation de la législation civile au Canada se complique du fait des recoupements juridiques fédéraux-provinciaux dans les domaines de justice criminelle et de santé mentale. La législation canadienne en matière de dangerosité semble donc nécessiter une solution made in Canada qui respectera les traditions canadiennes.

\section{ÉVALUATION CRITIQUE DE LA LÉGISLATION ET DE LA PRATIQUE COURANTES SUR LA DANGEROSITÉ AU CANADA}

La législation et la pratique courantes sur la dangerosité au Canada souffrent de plusieurs problèmes : réticence de plusieurs procureurs de la Couronne. particulièrement dans certaines provinces, à appliquer la loi ; l'usage apparent de la menace de classement comme contrevenant dangereux afin de négocier des peines réduites: l'apparent manque de considération des besoins en traitement des contrevenants classés dangereux.

Webster et Dickens (1983, p. 109) notent qu'on n'a fait aucune étude montrant que les contrevenants classés dangereux «faisaient preuve d'un comportement plus violent, plus dangereux, ou plus répétitif que celui des 
autres criminels". Quelles sont les différences ou ressemblances entre le petit nombre de contrevenants classés dangereux et le grand nombre de contrevenants violents et sexuels? Quelles caractéristiques rendent certains agresseurs admissibles, et d'autres non. au classement contrevenants dangereux? Quelles différences notables peuvent se manifester dans les catégories suivantes : crime grave; dossier judiciaire ; dossier psychiatrique : abus de drogues; violence physique ou sexuelle subie dans l'enfance ou exposition à une violence grave : race ou ethnie : apparence physique («bizarre» ou «normale»); classe sociale: antécédents familiaux ? Les contrevenants classés dangereux sont-ils vraiment différents des autres agresseurs sexuels violents, ou leur classement vient-il de leurs différences visibles et de la réponse du système judiciaire à ces différences?

Dans leur analyse de la législation canadienne de détention préventive, Wormith et Ruhl (1987, p. 425) arguent que «des différences régionales indiquent un sérieux problème de fiabilité et de consensus professionnel». À cet égard, on pourrait faire des recherches pour montrer comment et pourquoi les procureurs des différentes provinces désignent certains candidats comme contrevenants dangereux. Quelles sont les raisons du Québec pour ne pas se prévaloir du classement de contrevenant dangereux et pouquoi en fait-on un usage varié ailleurs? Des interviews avec les procureurs provinciaux pourraient aussi révéler l'étendue de l'utilisation de la menace de classement de contrevenant dangereux dans les négociations de peines réduites.

Finalement, on doit examiner l'évaluation des risques, la gestion des risques et les questions de traitement touchant les contrevenants dangereux dans le contexte des ressemblances ou des différences que ces contrevenants présentent par rapport au groupe plus nombreux des agresseurs sexuels violents.

\section{ÉVALUATION CRITIQUE DES PROPOSITIONS DU SOLLICITEUR GÉNÉRAL VISANT LES AGRESSEURS VIOLENTS À HAUT RISQUE}

Récemment, en réponse aux mouvements populaires privilégiant d'abord les drojts des victimes et de la société à se protéger, un nouveau modèle de protection communautaire est né. Dans un rapport sur le mouvement de protection communautaire de l'État de Washington. Scheingold et al. (1992, p. 17) prétendent que ces mouvements populaires «se basent idiosyncratiquement sur les réactions du public devant des crimes atroces» et que «ces êvénements extrêmes exagèrent les coûts sociaux du crime et en ignorent les complexités». Comparée à la législation de protection communautaire de l’État de Washington, la réforme canadienne présente certains avantages : 
(1) la capacité de s'appliquer à la fois aux contrevenants sexuels et aux contrevenants violents dont le crime n'est pas de nature sexuelle ;

(2) la capacité d'adopter avec flexibilité un éventail d'options postsentencielles (incluant des sentences déterminées et une supervision intensive en liberté) tenant compte des besoins de sécurité publique et de ceux de certains contrevenants ;

(3) la possibilité de libération conditionnelle.

De plus, contrairement aux lois d'Angleterre, du Pays de Galles et des Pays-Bas, un diagnostic de désordre mental ou de la personnalité n'est pas nécessaire pour classer un contrevenant dangereux. Il n'est pas question, non plus, de la responsabilité, partielle ou totale, de leur crime. Finalement. contrairement à Victoria, Australie. il n'y a pas d'exigence d'hospitalisation psychiatrique involontaire préalable.

Les propositions canadiennes ressemblent toutefois aux autres sur certains points. L'un des problèmes touchant les mesures post-détention pour les contrevenants purgeant déjà une sentence pour un crime précis est celui de double condamnation (double jeopardy). Actuellement, la demande d'enquête visant à classer un individu «contrevenant dangereux» doit se faire après la condamnation de cet individu pour un «crime grave contre la personne», mais avant le prononcé de la sentence. À l'heure actuelle, un individu ayant déjà reçu une sentence pour un crime ou une série de crimes ne peut recevoir une seconde sentence pour le même crime ou la même série de crimes. En cela. on respecte l'exigence fondamentale de la tradition juridique anglaise et anglo-américaine : une personne ne peut être placée en situation de double condamnation, c'est-à-dire subir deux procès et deux condamnations pour un seul crime ou une seule série de crimes (Sears. 1960-1961, p. 236).

En ce qui a trait aux propositions touchant les agresseurs à haut risque, l'exigence d'éviter la double condamnation n'est peut-être pas satistaite. Pour les contrevenants ayant purgé ou presque leur sentence pour un crime en particulier, la tenue d'une évaluation pour classement comme contrevenant dangereux et l'imposition d'un ordre de post-détention, alors qu'on a évalué les probabilités de délits futurs mais qu'aucun crime grave contre la personne n'a été commis, peut constituer une violation de la Charte des droits et libertés. Et ce problème se complique du fait que les clauses canadiennes touchant les contrevenants dangereux font partie du Code criminel et non du Code civil, comme dans l'État de Washington. En 1938, la Cour suprême des États-Unis a déclaré inconstitutionnelle la loi Criminal Sexual Psychopath du Michigan, adoptée en 1937 et faisant partie de son code criminel, sur la base, entre autres, de l'existence de la double condamnation (Petrunik, 1982, p. 231). Cette décision a mené à l'adoption du 
premier code civil sur les psychopathes sexuels, en Illinois, en 1938. Par la suite, aux États-Unis, les lois sur les psychopathes sexuels et agresseurs sexuels ont été adoptées dans le cadre du code civil. C'est aussi la raison pour laquelle l'État de Washington a adopté sa loi Sexually Violent Predators dans le cadre de son code civil.

La loi Community Protection de Victoria fait partie du code criminel, mais elle exige. dans le cas d'un ordre de protection communautaire de détention préventive, à la fois au moins un internement involontaire dans un hôpital psychiatrique et un diagnostic de troubles sérieux de la personnalité. Cette loi ressemble un peu aux clauses canadiennes sur les contrevenants mentalement déséquilibrés pour les personnes trouvées non coupables pour insanité ou incapacité à subir un procès. en ce sens que cette mesure vise à protéger la société contre une certaine catégorie de patients psychiatriques. La loi canadienne sur le Contrevenant dangereux, par ailleurs, même si elle demande une évaluation psychiatrique, n'exige pas d'hospitalisation psychiatrique préalable ni de diagnostic de trouble mental ou de la personnalité. En résumé, l'intention du Canada d'utiliser une désignation postsentencielle de contrevenant dangereux comme partie intégrante du Code criminel constitue plus clairement un cas de double condamnation que la loi civile de l'État de Washington ou la version de Victoria. La question de double condamnation devra se résoudre devant la Cour, si les propositions sont adoptées.

Un autre problème est celui des nouveaux pouvoirs que les propositions donnent aux autorités correctionnelles et au Conseil national des libérations conditionnelles dans le processus de désignation du contrevenant dangereux. Même si ces autorités sont déjà impliquées dans l'évaluation du risque de tous les contrevenants quant aux décisions touchant la détention et la libération, on doit évaluer soigneusement les effets des nouvelles législations sur la gestion des sentences des prisonniers. Afin d'obtenir plus de coopération, les autorités peuvent maintenir au-dessus de la tête des prisonniers, comme une épée de Damoclès, la possibilité d'un classement de contrevenant dangereux post-sentenciel. Quelles seraient les conséquences d'une telle menace? Les prisonniers seront-ils plus ou moins coopératifs avec les instances institutionnelles? Les contrevenants serontils plus ou moins enclins à modifier leur comportement?

Lafond (1993), dans une critique de la législation de l'État de Washington, indique déjà que certains agresseurs sexuels prisonniers dans l'État de Washington refuseraient les traitements. de crainte de fournir, pendant le traitement, des informations qui pourraient servir à les faire classer comme prédateurs sexuels violents. Lafond va même jusqu'à dire que la menace d'une détention post-sentencielle, sapant l'avantage de parti- 
ciper à un traitement ou un programme de prévention de rechute, peut accroître le risque pour la société.

Un troisième problème touche le développement d'une jurisprudence faisant l'équilibre entre. d'une part, les droits de l'agresseur et. d'autre part. ceux de la société à se protéger et ceux des victimes et de leurs familles a des réparations. Pepino (1993, p. 13) soutient que «la gestion du risque doit centrer son action sur l'agresseur, non sur le crime. et certainement pas sur la sentence ». D'après elle :

Les juges peinent encore sous un amas de précédents créés à l'époque où [...] [ils] entendaient des preuves d'honorabilité à la défense de l'accusé, mais aucune déclaration de la victime pour les contrebalancer.

Le modèle naissant de protection communautaire de gestion du risque semble toutefois entrer en conflit avec l'ancien modèle juridique de contrôle social, comme l'indiquent les critiques des défenseurs des libertés individuelles à l'endroit de la loi SVP de l'État de Washington et la loi Community Protection de Victoria. La gestion du risque dans l'intêrêt de la sécurité publique est-elle compatible avec les principes de justice individuelle, tels que stipulés dans la Charte des droits et libertés et autres déclarations de droits fondamentaux?

Le plus grand problème touchant à la fois les propositions du gouvernement conservateur sur la protection communautaire et la législation actuelle sur le contrevenant dangereux est que ceux-ci s'intéressent principalement à la dangerosité exceptionnelle, comme pathologie des agresseurs masculins qui attaquent les femmes et les enfants. Les récentes théories et recherches brossent un autre tableau.

Premièrement, la violence apparaît comme un problème envahissant se produisant dans un contexte familial ou dans le cadre de relations entre connaissances. Deuxièmement, la violence ne serait qu'occasionnellement le produit d'individus exceptionnellement pathologiques et le plus souvent le produit de structures sociales fondamentales et d'habitudes culturelles. Les pratiques de socialisation des sexes, les images de violence dans la culture populaire, l'inconstance de la discipline à la maison et à l'école et la commercialisation de la sexualité féminine auraient un impact important sur la société et contribueraient à la violence et aux agressions sexuelles.

\section{CONCLUSION}

L'histoire de la législation sur les contrevenants dangereux révèle une attention marquée pour certains contrevenants violents et sexuels dont plusieurs ne sont pas clairement plus dangereux que la plupart des autres contrevenants sexuels et violents. De plus, cette législation peut faire 
problème parce qu'elle s'attache davantage à la violence de quelques contrevenants prédateurs plutôt qu'à la violence quotidienne et routinière. Néanmoins, on peut croire que cette législation restera en place, peut-être avec des amendements, afin de mettre l'accent sur la protection communautaire.

Premièrement, malgré l'exagération des peurs du public. il y a effectivement des individus exceptionnels (Earl Shrine et Joseph Fredericks en sont de bons exemples) contre lesquels la société doit se protéger et pour qui les structures régulières de sentence et d'internement sont insuffisantes. Deuxièmement, les réactions du public (peur. colère, indignation) devant les crimes de violence sexuelle, particulièrement contre les enfants, sont à ce point fortes que toutes les mesures prises par le gouvernement pour éliminer de tels crimes, peu importe leur coût, recevront l'appui populaire. Les gouvernements qui accordent moins d'importance à la protection du public et plus d'importance aux droits des contrevenants et à leur réhabilitation risquent, dans le climat actuel, de s'aliéner leur base électorale. Pour ces raisons, je crois que la société continuera à s'éloigner du modèle clinique et du modèle juridique et privilégiera graduellement le modèle de protection communautaire.

\section{BIBLIOGRAPHIE}

ABEL, G. et ROULEAU, J. (1990), "The Nature and Extent of Sexual Assault ", in W. Marshall et al., (dir.), Handbook of Sexual Assault: Issues and Treatment of the Offender. New York, Plenum Press.

ABEL G, et al., (1987), "Self-Reported Sex Crimes of Non-incarcerated Paraphiliacs", Journal of Interpersonal Violence, vol. 2, pp. 3-25.

ANCEL, M. (1965), Social Defence : A Modern Approach to Criminal Problems, New York, Schocken.

ANTTILA, I. (1975), Incarceration for Crimes Never Committed, Helsinki, Research Institute of Legal Policy.

APPLEBY, T. (1992), «Plans to Release Pcdophile Put Pressure on Parole Board ", The Globe and Mail, 26 mai, pp. A1, A2.

BAKER, E. (1993), «Dangerousness, Rights, and Criminal Justice ", The Modern Law Review, vol. 56, pp. 528-247.

BELLAK, L. (1971), "The Need for Public Health Laws for Psychiatric Illness", American Journal of Public Health, vol. 61, pp. 119-121.

BERZINS, L. (1983), «Dangerous Offenders », rapport inédit, Ottawa, Ministry of the Solicitor General.

BEST, J. (1990), Threatened Children : Rhetoric and Concern About child Victims, Chicago, The University of Chicago Press.

BINDMAN, S. (1993), «Canadians Want High-Risk Offenders Identified, Poll Finds», The Citizen, 27 févricr, p. A3. 
BODINE, B. (1990) «Comment : Washington's New Violent Sexual Predator Commitment System : An Unconstitutional Law and an Unwise Policy Choice», University of Puget Sound Law Review, $\mathrm{n}^{\circ}$ 105, pp. 115-119.

BOERNER, D. (1992), "Confronting Violence : In the Act and in the Word», University of Puget Sound Law School, vol. 15, pp. 525-577.

BOERNER, D. (1993), «entrevue .

BLEECHMORE, J. (1975), * Towards a Rational Theory of Criminal Responsibility: The Psychopathic Offender", Melbourne University Law Review, vol. 10, Part Onc : May 1975, pp. 19-46; Part two : Sept, 1975, pp. 207-224.

BRICKMAN, J. et BRIËRE, J., "Incidence of Rape and Sexual Assault in an Urban Canadian Population ", International Journal of Women's Studies, 7, (May/June, 1984) $195-206$.

BROOKS, A. (1992), « The Constitutionality and Morality of Civilly Committing Violent Sexual Predators», University of Puget Sound Law Review, vol. 15, pp. 709 754.

BURICK, S. (1968), "An Analysis of the Illinois Sexually Dangerous Persons Act», Journal of Criminal Law, Criminology and Police Science, $\mathrm{n}^{\circ}$ 59, pp. 254-266.

CANADA. MINISTÈRE DU SOLLICITEUR GENERAL (1993), Rapport sur les recommandations préliminaires des groupes de travail sur les délinquants dangereux. Ottawa, le Ministère.

CANADA. COMITÉ DE LA RÉFORME PÉNALE ET CORRECTIONNELLE (1969), Rapport du Comité. Justice pénale et correction : un lien a forger. Ottawa, Imprimeur de la Reine.

CHANDLER, J. et ROSE, S., (1973), «The Constitutional Dilemna of a Person Predisposed to Criminal Behavior», Vanderbilt Law Review, vol. 26, pp. 69-103.

CIRALI, L. (1975), "The Law on the Boundary Line", International Journal of Offender Therapy and Comparative Criminology, vol. 22, pp. 80-90.

COCOZZA, J. et STEADMAN, H. (1976), « The Psychopath : The Failure of Psychiatric Predictions of Dangerousness: Clear and Convincing Evidence», Rutgers Law Review, vol. 29, pp. 1084-1161.

COCOZZA, J. et STEADMAN, H. (1978), «Prediction in Psychiatry : An Example of Misplaced Confidence in Experts», Social Problems, vol. 25, pp. 265-276.

COLlIGNON, T. et VAN DER MADE. R. (1943), La Loi belge de défense sociale à l'égard des anormaux et des délinquants d'habitude, Maison F. Larcier S.A.

CANADA. Comitố sur les infractions sexuelles à l'égard des enfanis (1984), Infractions sexuelles à l'égard des enfants. Rapport du conité. Otıawa, Approvisionnement et Services, 1984, vol. 1.

CONRAD, P. et SCHNEIDER, J. (1980), Deviance and Medicalization: From Badness ro Sickness, Toronto, C.V. Mosby.

CONTRACT RESEARCH CORPORATION, The Evaluation of Patuxent Institution : Final Report, Belmont, Mass, C. R. C.

DENMARK, MINISTRY OF JUSTICE-DEPARTMENT OF PRISONS, (1974, 1975), The Penal System of Denmark.

DERKS, F. C. H. (1993), et al., «Treatment and Security. The Dual Nature of Forensic Psychiatry ", Inernational Journal of Law and Psvchiatry, vol. 16, pp. 217-240. 
DIRECTION DE LA RECHERCHE ET DES STATISTIQUES. SERVICE CORRECTIONNEL DU CANADA (1993), La durée du risque de récidive chez les délinquants scxuels. Forum, 5, 2, pp. 8-10.

DIRECTION DE LA RECHERCHE ET DES STATISTIQUES. SERVICE CORRECTIONNEL DU CANADA (1991), Tout ce que vous avez toujours voulu savoir sur les délinquants sexuels fédéraux et davantage, Forum, 3, 3, pp. 3-6.

ENGLAND AND WALES (Department of Health and Social Security), (1987), Mental Health Act 1983 : Memorandum on Parts $I$ to VI, Viii and X, Londres, HMSO.

ENGLAND AND WALES, (1987), Mental Health Act 1983, Londres, HMSO.

ESSES, V. et WEBSTER, C. (1988), «Physical Attractiveness, Dangerousness and the Canadian Criminal Code», Journal of Applied Social Psychology, vol. 18, pp. 1017-1031.

EVENSEN, A. Social Defense in Norway, Washington : U.S. Department of Justice, NCJR, Microfiche, r.d., 1965.

FAIRALL, P. (1993), *Violent Offenders and Community Protection in Victoria. The Gary David Experience », Criminal Law Journal, vol 17, pp. 40-54.

FINKELHOR, D. et al. (1986), A Sourcebook on Child Sexual Abuse, Beverly Hills, Sage.

FOGEL, D. (1975), We are the Living Proof: The Justice Model of Corrections, Cincinnati, W. H. Anderson.

FOUCAULT, M. (1978), “About the Concept of the "Dangerous Individual" in 19th Century Legal Psychiatry International Journal of Law and Psychiatry, $\mathrm{n}^{\circ} 1$, pp. 1-19.

FUJIMOTO, B. (1992), «Sexual Violence, Sanity, and Safety : Constitutional Parameters for Involuntary Commitment of Sex Offenders ", University of Puget Sound Law Review, vol. 15, pp. 879-911.

FURBY, L. et al. (1989), «Sex Offender Recividism : A Review», Psychological Bulletin, $\mathrm{n}^{\circ} 105$, pp. 3-30.

GLEB, G. (1991), «Comment, Washington's Sexually Violent Predator Law: The Need to Need to Bar Unreliable Psychiatric Predictions of Dangerousness from Civil Commitment Proceedings », U.C.L.A. Law Review, vol. 39, pp. 213-250.

GRABOWSKI, J. (1988), "Comment, The Illinois Sexually Dangcrous Person's Act: An Examination of a Statute in Need of Change $»$ Southern Hinois Universitv Law Journal, vol. 12, pp. 437-487.

GREENLEES, L. (1991), « Note, Washington State's Sexually Violent Predators Act : Model or Mistake", American Criminal La Review, vol. 29, pp. 107-132.

GROTH, A.N. et al. (1982), «Undetected Recidivism Among Rapists and Child Molesters ", Crime and Delinquency, pp. 450-458.

HAKEEM, M. (1958), «A Critique of the Psychiatric Approach to Crime and Correclions ", Law and Contemporary Problems, vol. 23, pp. 650-682.

HANS ARD, House of Commons Debates 4th Session, 20th Parliament, June 14, 1948 . vol. 5, pp. 5195-5200.

HANSON, R. K. (1990) « The Psychological Impact of Sexual Assault on Women and Children : A Revicw», Annals of Sex Reseurch, vol. 3, pp. 187-232. 
HANSON, R. K., SCOTT, H. et STEFFY, R. (1993), « A Comparison of Child Molesters, and Non Sexual Criminals : Risk Predictors and Long Term Recidivism», article inédit.

HARE, R. (1986), "Twenty Years of Experience with the Cleckley Psychopath " in W. Reid et al. (dir.), Unmasking the Psychopath: Anti-Social Personality and Relared Syndromes, New York, W.W. Norton, pp. 3-27.

HARRIS, G. et RICE, M. (1994), " Psychopaths : "Is a Therapeutic Community" Therapeutic?", Peneranguishene Mental Health Centre Research Reports, VIII, 31 pages.

HARRIS, G., RICE, M., et CORMIER, C. (1991), « Psychopathy and Violent Recidivism», Law and Human Behaviour, vol. 15, pp. 625-637.

HART, S. et al. (1988), «Performance of Male Psychopaths Following Conditional Release From Prison», Journal of Consulting and Clinical Psychology, vol. 56, pp. 227-232.

HARVARD LAW REVIEW, (1975), «The Constitutionality of Statutes Permitting Increased Sentences for Habitual or Dangerous Criminals ", vol 89, pp. 356-386.

HUDSON, K. (1993), «Keep Sexual Predators Behind Bars Jury Urges », Toronto Star, 23 janvier, pp. A1, A2.

KENDALL-TACKETT K. et al. (1993) «Impact of Sexual Abuse on Children : A Review and Synthesis of Recent Empirical Studies», Psychological Bulletin, vol. 113, pp. 164-180.

KOENRAADT, F. (1990), «The Forensic Psychologist in Dutch New Legislation and in Forensic Residential Assessment", article présenté à la seconde European Conference on Law and Psychology, Nuremberg, 13-15 septembre.

KITTRIE, N. (1971), The Right To Be Different, Baltimore, Penguin Books.

KOZOL, H. et al. (1972), * The Diagnosis and Treatment of Dangerousness", Crime and Delinquency, $\mathrm{n}^{\circ}$ 18, pp. 371-392.

LAFOND, J. (1992), «Washington's Sexually Violent Predator Law : A Deliberate Misuse of the Therapeutic State for Social Control ", University of Puget Sound Law Review, 15, pp. 655-703.

LAFOND, J. (1993) « Personal Interview ».

LAFOND, J. ct KAGAN, K. (1992), «In re the Detention of André Brigham Young and Vance Cunningham, Brief of Amicus Curiac in support of appellants», Tacoma, American Civil Liberties Union of Washington.

LAWS, R. (1989), Relapse Prevention with Sexual Offenders, New York. Guilford.

LIZOTTE, A. (1985), «The Uniqueness of Rape : Reporting Assaultive Violence to the Police", Crime and Delinquency, vol. 31, pp. 169-190.

LOWMAN, J. et al., (1986). Regulating Sex : An Anthology on the Badgley and Fraser Reports Burnaby B.C., Simon Fraser University.

MARSHALL, W. et BARBAREE, H. (1988), «The Long-Term Evaluation of a Behavioural Treatment Program for Child Molesters », Behavior Research Therapy, $n^{\circ}$ 26, pp. 499-511.

MARSHALL, W. et BARBAREE, H, (dir), (1990), Handbook of Sexucl Assault: Issues. Theories and Treatment of the Offender, New York, Plenum Press. pp. 363-385. 
MARSHALL, W. et BARRETT, S. (1990), Criminal Neglect: Why Sex Offenders Go Free, Toronto, Doubleday.

MARSHALL, P. et VAILLANCOURT, M. (co-présentés), (1993), Un nouvel horizon : Éliminer la violence, atteindre l'égalité. Rapport final du comité canadien sur la violence faite aux femmes. Ottawa, Ministère des Approvisionnement et Services.

MATHIESEN, T. (1965), The Defences of the Weak : A Sociological Study of a Norwegian Correctional Institution, Londres, Tavistack Publications.

MCGARRY, A. L. et PARKER. L. L., (1974) «Massachusetts Operation Baxstrom : A Follow-up ", Massachusetts Journal of Mental health, $\mathrm{n}^{\circ} 4$, pp. 27-41.

MCGEE, R. (1978), «California's New Determinate Sentencing Act ", Federal Probation, $\mathrm{n}^{\circ} 42$, pp. 3-10.

MINISTRY OF THE SOLICITOR-GENERAL (1993a), « Proposals to Amend the Corrections and Conditional Release Act and the Criminal Code », Ottawa.

MINISTRY OF THE SOLICITOR-GENERAL (1993b), «Proposals to Amend the Corrections and Conditional Release Act, the Criminal Code, the Prisons and reformatories Act and the Transfer of Offenders Act», Ottawa.

MONAHAN, JOHN (1988), "Risk Assessment of Violence Among the Mentally Disordered : Generating Useful Knowledge ", International Journal of Law and Psychiatry, vol. 11, pp. 249-257.

MONAHAN, J. (1984), «The Prediction of Violent Behavior : Toward a Second Generation of Theory and Policy", American Journal of Psychiatry, vol 10, $\mathrm{n}^{\circ} 41$, pp. 10-15.

MONAHAN, J. (1981), Predicting Violent Behaviour: An Assessment of Clinical Techniques, Beverly Hills, Sage.

MOYER, L. (1974), «The Mentally Abnormal Offender in Sweden : an Overview and Comparisons with American Law\%, American Journal of Comparative Law, vol. 22, pp. 71-106.

NANGLE, O. (1976), «Dangerousness, Reasonable Doubt and Preconviction Psychopath Legislation», Southern Illinois University Law Journal, $\mathrm{n}^{\circ}$ 1, pp. 218-236.

THE NETHERLANDS, PRISON SERVICE, Detention at the Government's Pleasure: Treatment of Criminal Psychopaths in the Netherlands, La Hage, Central Recruitment and Training Institute of the Prison Service and the Care of Criminal Psychopaths Service.

THE NETHERLANDS (MINISTRY OF JUSTICE) (1991), TBS : A Special Measure Within the Criminal Code, Leyde, Zorn Publishing.

OLIVER, A. (1982), «The Sex Olfender: Lessous from the California Experience», International Journal of Law and Psychiatry, vol. 5, pp. 403-411.

ONTARIO, MINISTRY OF THE SOLICITOR-GENERAL, Office of the Chicf Coroner (1993), Inquest into the Death of Christopher Stephenson : Verdict of the Jury, Toronto.

PEAY, J. (1988), "Offenders Suffering From Psychopathic Disorder: The Rise and Demise of a Consultation Document», British Journal of Criminology, $n^{\circ} 28$, Pp. $67-81$.

PEPINO, N. J. (1993), «Managing Risk-Whose Problem is it Anyway?». Forum on Corrections Research, vol. 5, pp. 12-13. 
PETRUNIK, M. (1982), "The Politics of Dangerousness", International Journal of Law and Psychiatry, no 5, pp. 225-253.

PITHERS, W. (1990). «Relapse Prevention With Sexual Aggressors », in W. Marshall, D. Laws et H. Barbaree (dir.), Handbook of Sexual Assault, New York, Plenum Press, pp. 343-361.

POLK, K. (1985), «A Comparative Analysis of Attrition of Rape Cases», British Journal of Criminology, vol. 25, pp. 28-284.

POS, R. et al., (1987), Dangerous Offender Hearings in British Coltumbia: A Preliminary Report of a Review of the First Twenty-One Cases Under the 1977 Legislation. Vancouver, Forensic Psychiatry Services Commission.

QUINSEY, V. (1992), «Review of Sexual Predator Programs : Community Protection Research Project», Olympia, Washington: Washington State Institute for Public Policy.

RICHARDSON, M. (1993), "Chief Wants Sex Offender Alerts", The Citizen, 4 février, p. B1.

ROBINSON, D. (1989), "Research on Sex Offenders : What Do We Know? », Forum on Corrections Research, vol. 1, pp. 12-20.

ROGERS, D. (1993a), «Naming Ottenders Violates Rights Expert Says», The Citizen, 16 fevrier, p. A1.

ROGERS, D. (1993b), «Nepean Police To Release Names of High-Risk Offenders », The Citizen, 13 février.

ROSS, K. P. et HOCHBERG, J. M. (1978), «Constitutional Challenges to the commitment and Release Procedures Under Massachusetts General Laws Chapter 123A, The "Sexually Dangerous Persons" Act " New England Journal on Prison Law, vol, 4, pp. 253-308.

REIBER, R. et VETTER, H. (dir.) (1978), The Psychological Foundations of Criminal Justice, vol. 1, New York, The John Jay Press.

SANSONE, J. (1976), Sentencing, Corrections and Special Treatment Services in Sweden. Denmark and the Netherlands, Hartford, Connecticut, Hartford Institute of Criminal and Social Justice.

SARICK, L. (1993), «Stephensun Inquest: A Predator Observed ", Toronto Globe and Mail, 23 janvier, p. DI.

SCHEINGOLD, S. (1984), The Politics of Law and Order, New York, Longman.

SCHEINGOLD, S. et al., (1992), «Republican Criminology and Victim Advocacy: Washington State's Sexual Predalor Legistation», article présenté a l'American Society of Criminology Mectings, New Orlcans, novembre 1992.

SCHEINGOLD, S. et al. (1992), "The Politics of Sexual Psychopalliy: Washington State's Sexual Predator legislation ", University of Puget Sound Law Review, vol. 15, pp. 809-820.

SEARS, R. (1960-1961), «Illinois Double Jeopardy Act : An Empty Gesture», Journal of Criminal Law. Criminology and Potitical Science, vol. 51, pp. 236-240.

SERRILL, M. (1977a), «Profilc/Denmark», Corrections Magazine, pp. 23-42.

SERRILL, M. (1977b), «Profilc/Sweden », Corrections Magazine, pp. 11-14, 18-36.

SERRILL, M. (1977c), «Determinale Sentencing. The History, The Theory, The Debate», Corrections Magazine. pp. 1-30, 33-68. 
SHORE, J. (1984), «An Evaluation of Canada's Dangerous Oflender Legislation», Les Cahiers de druit, vol 25, pp. 441-426.

STATE OF WASHINGTON (1989), Governor's Task Force on Community Prevention, Olympia, Washington. Dept. of Health and Social Services.

STATE OF WASHINGTON (1991), Sexually Violent Predators Stature, Revised Code of Washington, chapter 71.09 (Supp. 1990-91).

STONE, A. (1975), Mental Health and Law : A System in Transition, Rockville, Maryland, National Institute on Mental Health.

SLEFFEL, L. (1977). The Law and the Dangerous Criminal: Statutory Attempts at Definition and Control, Lexington, Lexington Books.

SOLLICITEUR GÉNÉRAL DU CANADA (1985), «Les femmes victimes d'actes criminels ", Canadian Urban victimization Survey Bulletin, $\mathrm{n}^{\circ} 4$.

STEADMAN, H. (1972), "The Psychiatrist as a Conservative Agent of Social Control», Social Problems, n' 20, pp. 263-272.

STEADMAN, H. (1976), "Predicting Dangerousness" in D. Madden et J. Lion, Rage. Hate, Assault and Other Forms of Violence, New York, Spectrum Publications.

STEADMAN, H. (1978), "Psychiatry, Dangcrousness and the Repetitively Violent Offender ", Journal of Criminal Law and Criminology, 69, pp. 226-232.

SUMMERS, D. (1991), «In Re. The Personal Restraint of Andre Bringham Young and Vance Cunningham, Brief of Amicus Curiae in Support of Petitioners» Seattle, Washington State Psychiatric Association.

SUTHERLAND, E. (1973), "The Sexual Psychopath Laws», Journal of Criminal Law and Criminology, 40, (Jan-Feb, 1950a) pp. 443-454 reprinted in K. Schuessler, ed, Edwin H. Sutherland on Analyzing Crime, Chicago, University of Chicago Press.

SUTHERLAND, E. (1975), "The Diffusion of Sexual Psychopath Laws", American Journal of Sociology, 56, (Sept. 1950b) pp. 142-148 reprinted in F. Davis et R. Stivers, (dir.), The Collective Definition of Deviance, New York, The Free Press, pp. 281-289.

SVENDSEN. B. (1977), «Declining Interest in Forensic Psychiatry : Recent Developments in Denmark "The Bulletin of the American Academy of Psychiatry and the Law, 1, pp. 20-28.

SWANSON, A. (1960-1961), "Sexual Psychopath Statutes: Summary and Analysis" Journal of Criminal Law. Criminology and Political Science, 51, pp. 215-236.

SZASZ, T. (1975), "The Expulsion of Evil" in F. J. Davis and R. Stivers, (dir.), The Collective Definition of Deviance. New York, The Free Press, pp. 60-72.

SZASZ, T. (1965), Psvchiatric Jusrice, New York, MacMillan.

SZASZ, T. (1977), Psychiatric Slavery, New York, The Free Press.

TAPPAN, P. (1950), «Sex Offender Laws and Their Administration"Federal Probation, 14, pp. 32-37.

TEMBORIUS, E. (1977), "Sexually Dangerous Persons Act - Burden of Proof Sexually Dangerousness Must Be Established Beyond a Reasonahle Doubt Pcople v. Pembrock, 62 Ill. 2nd 317, 342 N.E 2nd 28 (1976)», De Paul Law Review, 26, pp. 392-400.

TENNEY, C. W. (1962), "Scx, Sanity and Slupidity in Massachussets» Boston Universiry Law Review, XLII, pp. 2-31. 
THORNBERRY, T. et JACOBY, J. (1977), * The Released Criminally Insane Offender : Social and Psychological Adjustment in C. R. Huff, (dir.), m, Contemporary Corrections: Social Control and Conflict, Beverly Hills, California, Sage Publication, pp. 124-139.

TIGGES, L. C. M. (1990), «Exit Procedures of Forensic Hospitals in the Netherlands : Current Problems and Future Prospects» papier présenté au the XVIth International Congress on Law and Mental Health, Toronto.

TUlkenS, F. et DIGNEFFE, F. (1979), «La Notion de Dangerosité dans la Politique Criminclle en Europe Occidentale" présenté au Colloque Intemational en l'honncur du $50^{e}$ anniversaire de l'École de criminologie, Université Catholique de Louvain, Louvain-la-Neuve, Belgique, mai 22-25.

VAN EMMERIK, J.(1990), «The TBS Order : Treatment Results with Mentally Disordered Delinquents in the Netherlands», papier présenté l'International Congress on Law and Mental Health, Toronto, June.

VICTORIA, Australia, Attorney-General's department, (1990-1991), * Community Prolection Act 1990 and Amendment, 1991 », Melbourne, A.V. North, Government Printer.

VICTORIA, Australia, Attorney-General's Department (1992), «Drafts Proposals for a Community Protection (violent offenders) Bill », Melbourne, A.V. North, Government Printer.

VIENNEAU, D. (1993), "MP's Deny Sex Offenders Release» The Ottawa Citizen (feb. 11)

WALD, P. et FRIEDMAN, P. (1978), «The Politics of Mental Health Advocacy in the United States * International Journal of Law and Psychiatry, vol. 1, pp. 137-152.

WEBSTER, C. (1990), «Prediction of Dangerousness Polemic , Revue canadienne de criminologie, 32, pp. 191-196.

WEBSTER, C. (1990), «The Clinical Prediction of Dangerousness » in N. Hilton, M. Jackson et C. Webster, (dir.), Clinical Criminology : Theory, Research, and Practice, Toronto, Canadian Scholars Press, pp. 555-574.

WEBSTER, C. et DICKENS, B. (1983), Deciding Dangerousness Policy : Alternatives for Dangerous Offenders, Onawa, Department of Justice.

WISBERG, D. K. (1984), «The 'Discovery' of Sexual Abuse : Experts' Role in Legal Policy Formulation », U. C. Davis Law Review, 18, pp. 1-57.

WERLINDER, H. (1978), «Psychopathy : A History of the Concepts », Uppsala Studies in Education, vol. 6, Uppsala, Acta Universitatis Upsaliensis.

WESTON, D. (1993), «Interview With David Weston, Superintendent, Special Commitment Center $*$, Monroe. Washington, Feb. 27.

WETTSTEIN, R. (1992), \&A Psychiatric Perspective on Washington's Sexually Violent Predators Statute m. University of Puget Sound Law Review, 15, pp. 597-633.

WORMITH, S. et BORZECKI, M. (1987), «A Survey of Treatment Programs for Scx Offenders in North America ", Canadian Psychology, 28, pp. 30-44.

WORMITH, S. et RUHL, M. (1986), «Preventive Detention in Canada », Journal of Interpersonal Violence. 1, pp. 399-430.

WRIGHT, R. (1984), "A Note on the Attrition of Rape Cases", Brifish Jourmal of Criminologv, 24, pp. 399-400.

ZAGARIS, B. (1977), «The Finnish Penal System : Recent Reforms», New linglond Journal on Prison Law, vol. 3, pp. 437-487. 